\title{
Star forming galaxies in the AKARI deep field south: identifications and spectral energy distributions *
}

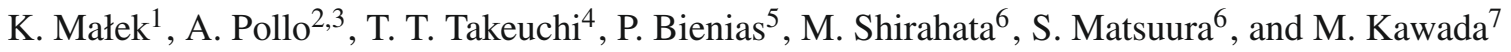 \\ ${ }^{1}$ Center for Theoretical Physics of the Polish Academy of Sciences, Al. Lotników 32/46, 02-668 Warsaw, Poland \\ e-mail:malek@cft.edu.pl \\ 2 The Andrzej Sołtan Institute for Nuclear Studies, ul. Hoża 69, 00-681 Warsaw, Poland \\ 3 The Astronomical Observatory of the Jagiellonian University, ul. Orla 171, 30-244 Kraków, Poland \\ 4 Institute for Advanced Research, Nagoya University, Furo-cho, Chikusa-ku, Nagoya 464-8601, Japan \\ ${ }^{5}$ College of Inter-Faculty Individual Studies in Mathematics and Natural Sciences, University of Warsaw, ul. Żwirki i Wigury 93, \\ 02-089 Warsaw, Poland \\ ${ }^{6}$ Institute of Space and Astronautical Science, JAXA, 3-1-1 Yoshinodai, Sagamihara, Kanagawa 229-8510, Japan \\ 7 Division of Particle and Astrophysical Science, Nagoya University, Furo-cho, Chikusa-ku, Nagoya 464-8602, Japan \\ Received 7 October 2009 / Accepted 24 November 2009
}

\section{ABSTRACT}

\begin{abstract}
Aims. We investigate the nature and properties of far-infrared (FIR) sources in the AKARI deep field south (ADF-S). Methods. We performed an extensive search for the counterparts to 1000 ADF-S objects brighter than 0.0301 Jy in the WIDE-S $(90 \mu \mathrm{m})$ AKARI band in the public databases (NED and SIMBAD). We analyzed the properties of the resulting sample: statistics of the identified objects, quality of position determination of the ADF-S sources, their number counts, redshift distribution, and comparison of morphological types, when the corresponding information was available. We performed a simplifield analysis of the clustering properties of the ADF-S sources and compliled spectral energy distributions (SEDs) of objects with the highest quality photometry, using three different models.

Results. Among 1000 investigated ADF-S sources, 545 were identified with sources at other wavelengths in public databases. From them, 518 are known galaxies and 343 of these were previously known as infra-red sources. Among the remaining sources, there are two quasars and both infrared and radio sources of unknown origin. Among six stellar identifications, at least five are probably the effect of contamination. We measured the redshifts of 48 extragalactic objects and determined the morphological types of 77 galaxies. We present SED models of 47 sources with sufficiently good photometric data.

Conclusions. We conclude that the bright FIR point sources observed in the ADF-S are mostly nearby galaxies. Their properties are very similar to the properties of the local population of optically bright galaxies, except for unusually high ratio of peculiar or interacting objects and a lower percentage of elliptical galaxies. The percentage of lenticular galaxies is the same as in the optically bright population, which suggests that galaxies of this type may frequently contain a significant amount of cool dust. It is possible that source confusion plays a significant role in more than $34 \%$ of measurements. The SEDs correspond to a variety of galaxy types, from very actively star forming to very quiescent. The AKARI long wavelength bands data have enabled us to determine for the first time that these galaxies are objects with very cool dust.
\end{abstract}

Key words. surveys - Galaxy: fundamental parameters - galaxies: evolution - infrared: galaxies

\section{Introduction}

Active star formation (SF) is related to heavy element production between the birth and death of stars. Several of the heavy elements produced by stars become substantially depleted inside dust grains. These dust grains in galaxies tend to absorb ultraviolet (UV) light, emitted by young stars, and re-emit it in the far infrared (FIR). There is an extreme category of galaxies that contains a large amount of dust and is extremely luminous at FIR and submillimeter (submm) wavelengths. Heavily hidden $\mathrm{SF}$ is understood to exist in these galaxies.

By examining the luminosity functions (LFs) at UV and FIR from GALEX and IRAS/Spitzer, Takeuchi et al. (2005a) demonstrated that the FIR LF evolves far more significantly than that

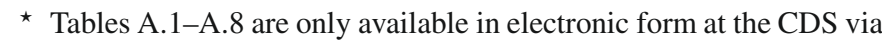
anonymous ftp to cdsarc.u-strasbg.fr (130.79.128.5) or via http://cdsweb.u-strasbg.fr/cgi-bin/qcat?J/A+A/514/A11 of UV, although both evolve very strongly. This indicates that the fraction of hidden SF rapidly increases to higher redshifts of $z<1$. Another important observable closely related to the dust emission from galaxies is the cosmic IR background (CIB). Takeuchi et al. (2006) constructed the IR spectral energy distribution (SED) of the local Universe. The energy emitted in the IR is $25-30 \%$ of the total energy budget. In contrast, the IR (from near/mid-IR to millimeter) contribution is roughly (or even more than) half of the CIB spectrum (e.g., Dole et al. 2006). This also suggests a strong evolution of the IR contribution to the cosmic SED in the Universe.

Understanding the radiative physics of dust is therefore a fundamental task in obtaining an unbiased view of the cosmic SF history. Exploring the evolution of galaxy SEDs at each epoch is particulary important to establishing a unified view of the SF history. The first step is to determine the properties of local galaxies. A vast amount of new information about the IR universe was 
provided by IRAS (e.g., Soifer et al. 1987), followed by MSX (e.g., Egan et al. 2003), ISO (e.g., Genzel \& Cesarsky 2000; Verma et al. 2005), and Spitzer (e.g., Soifer et al. 2008).

After IRAS, a Japanese IR satellite, AKARI ("akari" means a "light" in Japanese), performed an all-sky survey (Murakami et al. 2007) and various smaller but deeper surveys at different IR wavelengths. In particular, with the aid of the FarInfrared Surveyor (FIS: Kawada et al. 2007), observations in four FIR bands were possible. Among the observed fields, the lowest Galactic cirrus emission density region close to the South Ecliptic Pole was selected for observations because it can provide the highest quality FIR extragalactic image of the Universe. This field is referred to as the AKARI deep field south (ADF-S). This survey is unique in having continuous wavelength coverage with four photometric bands $(65,90,140$, and $160 \mu \mathrm{m})$ mapped over a wide area ( $\sim 12$ square degrees). To $\sim 20 \mathrm{mJy}$ at $90 \mu \mathrm{m} 2268$ infrared sources were detected, and infrared colors for about 400 of these were measured.

With the advent of AKARI surveys, a new generation of large databases of the Local Universe will become available. In this paper, we present the first results of our cross identification (cross-ID) of sources in the ADF-S. After describing the process of identifying ADF-S objects, we discuss their statistical properties. We then indicate the UV-optical-FIR SEDs of selected nearby star-forming galaxies in our sample with the highest quality photometric data. We then present our analysis of these SEDs performed by fitting a few simple models of dust emission from galaxies.

The paper is organized as follows: in Sect. 2, we present the data. Section 3 describes a basic analysis of the properties of identified sources, their distribution on the sky, and the quality of the catalog and possible biases, e.g., related to the source confusion. We then discuss the statistical properties of the obtained sample, e.g., the number counts, redshift distribution, galaxy morphologies, and other properties. In Sect. 4 we present the SEDs of the identified galaxies and we attempt to model the galaxy dust emission. We present our conclusions in Sect. 5.

\section{The data}

We cross-identified the ADF-S point source catalog (based on $90 \mu \mathrm{m}$ ) with publicly available databases, mainly the SIMBAD ${ }^{1}$ and $\mathrm{NED}^{2}$. We performed this search in two stages: first for 500 ADF-S sources brighter than $0.0482 \mu \mathrm{Jy}$ in the WIDE$S$ AKARI band $(90 \mu \mathrm{m})$, which corresponds roughly to our $\sim 10 \sigma$ detection, and then for an additional 500 ADF-S sources, brighter than $0.0301 \mu \mathrm{Jy}$ in the WIDE-S band, which corresponds approximately to our $\sim 6 \sigma$ detection. In the following sections, we refer to these two data sets as $10 \sigma$ and $6 \sigma$ catalogs. We present the properties of sources in these catalogs.

The search for counterparts was performed within the radius of $40^{\prime \prime}$ around each source. The ADF-S images were obtained using the slow-scan mode of FIS. The synthesized point spread functions (PSFs) of the slow-scanned image were presented and examined extensively in Shirahata et al. (2009). They showed that the PSF at each band is represented well by a "doubleGaussian profile", i.e., a superposition of two 2-dimensional Gaussian profiles with different standard deviations. The standard deviations of the narrower component are $32^{\prime \prime} \pm 1^{\prime \prime}$ (for the $65 \mu \mathrm{m}$ band), $30^{\prime \prime} \pm 1^{\prime \prime}$ (the $90 \mu \mathrm{m}$ band), $41^{\prime \prime} \pm 1^{\prime \prime}$ (the $140 \mu \mathrm{m}$ band), and $38^{\prime \prime} \pm 1^{\prime \prime}$ (the $160 \mu \mathrm{m}$ band). They also demonstrated

\footnotetext{
${ }_{1}$ URL: http://simbad.u-strasbg.fr/simbad/

2 URL: http://nedwww.ipac.caltech.edu/
}

that approximately $80 \%$ of the flux power is included in this component. Hence, this size can be practically regarded as a reasonable counterpart search radius. In the case of ADF-S, nearby galaxies have more extended profiles than a simple point source, and the reduction method and scan speed are slightly different from the early PSF analysis. In addition, we used all four FIS bands. Hence, we chose the largest size $40^{\prime \prime}$ as the tolerance radius in our counterpart search.

For sources from the $10 \sigma$ catalog within the search radii, we found in total 500 counterparts, corresponding to 330 ADF-S sources. Among them, there are two cases in which two ADF-S sources correspond to the same counterpart (one star and one galaxy). For 170 sources (34\%), no counterparts were found. For 208 sources (42\%), there is one possible counterpart, 114 sources $(23 \%)$ have two or three counterparts, and for 8 sources (1.6\%) more than 3 possible counterparts were found.

Extending the identification process to the $6 \sigma$ level, we found 284 more counterparts, corresponding to 215 sources fainter than $0.0482 \mu \mathrm{Jy}$ and brighter then $0.0301 \mu \mathrm{Jy}$ in the WIDE-S band. Among them were 49 cases (10\% of this fainter part of the sample) of a double and 10 cases (2\%) of a triple counterpart. Two sources correspond to the same counterpart (Seyfert-1 galaxy located at at $z \sim 0.24$ ). One of the sources corresponds to an extragalactic X-ray source that is related to a starburst galaxy NGC 1705, identified as the fourth brightest source in the $10 \sigma$ sample. For 156 sources (31\%), we found only one counterpart. No counterparts were found for 285 sources (57\%). Thus, the probability of finding a counterpart decreases with the $90 \mu \mathrm{m}$ brightness of sources by a factor of 1.5 . However, at the same time the probability of finding a source with multiple counterparts decreases much faster, by a factor of 2 . There are two possible reasons for this behavior: 1) In ADF-S data, a source confusion would give a more significant contribution to the flux of the brighter sources. This is usually not the case (e.g., Takeuchi \& Ishii 2004), but because of the special way of image construction in the ADF-S data, the confusion effect might play some role. 2) Interaction between physically close galaxies (and, consequently, on the sky) may increase their intrinsic IR luminosity significantly. The true situation would be a mixture of these two effects.

For the purpose of this work, we assume, unless specified otherwise, that the most nearby counterparts of ADF-S sources are real ones. However, as stated above, we are aware that the source confusion plays an important role at least in some of measurements of the multiply identified sources. We try to address this issue in Sect. 3.7.

\section{Basic analysis}

\subsection{Accuracy of the position determination of the ADF-S sources}

As shown in Fig. 1, the angular separation between the ADF-S source and its counterpart is smaller than $20^{\prime \prime}$ in most cases. This suggests that the true resolution of the ADF-S map corresponds roughly to the pixel size of the FIS detector. It is plausible that the more distant identifications are caused by the contamination. However, since they are few in number, their presence should not affect the quality of our sample and we decided to retain our original criterion.

A positional scatter map, shown in Fig. 2, displays a small but systematic bias of $\sim 4^{\prime \prime}$ in the declination of the ADF-S positions with respect to their counterparts. The dependence of a positional deviation on the right ascension $\alpha$ and declination $\delta$ 
K. Małek et al.: Star forming galaxies in the ADF-S

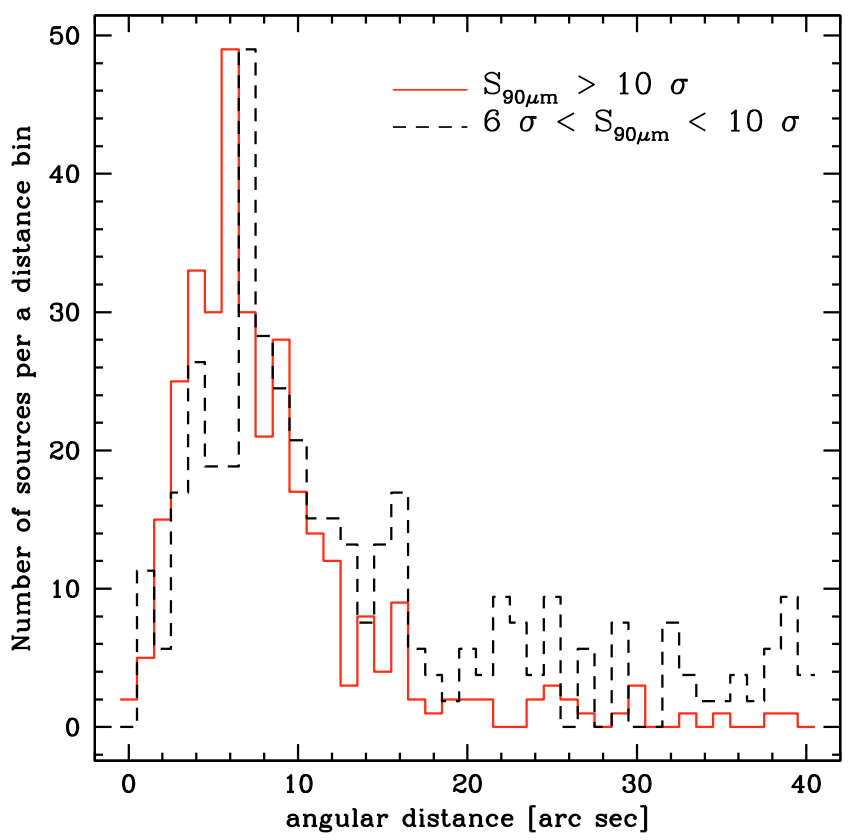

Fig. 1. The distribution of the angular deviations of the nearest counterparts from the ADF-S sources. A solid line corresponds to the $10 \sigma$ catalog, while a dashed line corresponds to the faint part of the $6 \sigma$ sample. In both cases, most of the identified sources have counterparts closer than $20^{\prime \prime}$. The precision of the position determination decreases with the source flux density - for the $10 \sigma$ sample, the median value of the angular distance of the source from a counterpart is 6.9 arcsec $(6.45$ below $20^{\prime \prime}$ ), while for the faint part of the $6 \sigma$ sample, the median value of angular distance of the source from a counterpart is $9.7 \operatorname{arcsec}(8.2 \mathrm{be}-$ low $20^{\prime \prime}$ ). The identifications at angular distance $>20^{\prime \prime}$ might be chance coincidences.

as a function of $\alpha$ and $\delta$ is presented in the four panels of Fig. 3 . We observe that the bias of the position determination depends on the source position, being strongest in the south-west part of the field. However, we note that in all cases the bias is much smaller than the uncertainty in the determined position caused by the AKARI FIS detector resolution.

The parameters of the the optimal rms fits to the data in Fig. 3 are listed in Table 1. The results are presented both for the 330 identified sources from the $10 \sigma$ catalog and for all 545 identified sources from the full $6 \sigma$ sample. We observe that including the fainter sources in the sample increases the observed scatter, although, at the same time it reduces the bias in the determination of $\alpha$ and its dependence on the source position in the ADF-S. Thus, we should be aware that the positions of the brightest ADF-S sources are the most biased, even if the bias itself remains small. This bias may be produced by the complex method of source extraction from the slow-scan data in the ADF-S (for details, see Shirahata et al., in prep.).

\subsection{Classification of objects identified in the ADF-S}

In the $10 \sigma$ catalog, among 330 identified objects, 314 are known galaxies (one appearing twice). Of these galaxies, 173 (55\%) were previously observed in the infrared (either by IRAS or 2MASS) but $141(45 \%)$ of them were not known previously to be infrared sources. The remaining 16 objects include radio and infrared sources of unknown nature, five stars and one quasar at $z=1.053$. However, the stars in this sample belong to a sparse group of objects at the distance of a counterpart from the

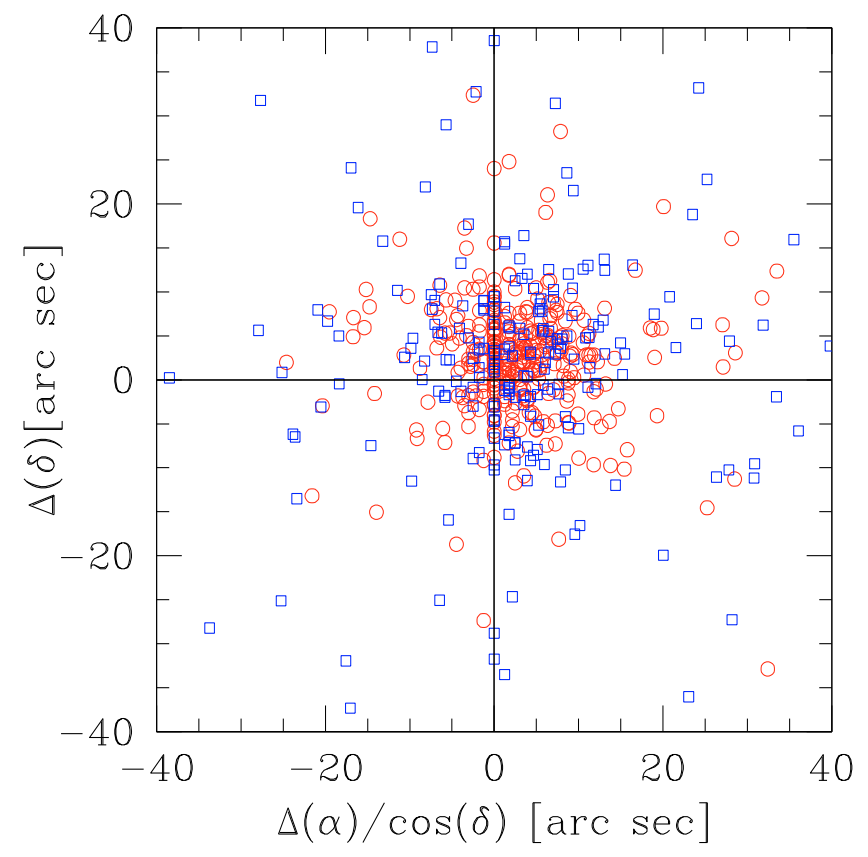

Fig. 2. Scatter plot of the deviation of counterparts from the ADF-S sources in right ascension and declination. Open circles denote sources from the $10 \sigma$ catalog, and open squares - remaining objects from the faint part of the $6 \sigma$ catalog. We can observe a small but systematic bias, which is similar for brighter and fainter parts of the sample, even if the scatter for the fainter objects is larger.

ADF-S object close to 40". A careful examination indicated that they are probably falsely identified because of the contamination (Fukagawa, private communication).

In the fainter half of the $6 \sigma$ catalog, i.e., fainter than $10 \sigma$, among 215 identified sources, 205 are known galaxies (one appearing twice). However, only 32 (16\%) of them were previously known as infrared sources. The remaining 173 galaxies are observed in infrared for the first time. Among the remaining objects, there are 7 radio sources of unknown nature, one quasar at $z \sim 1.23$, one X-ray source related to the starburst galaxy NGC 1705, which is the fourth brightest source in our $10 \sigma$ data set, and one double stellar system of $B \sim 6$. A summary of the properties of identified sources is given in Table 2 .

The data related to the nearest counterparts of the ADF-S sources are summarized in tables that are available from the database of CDS (Centre de Données astronomiques de Strasbourg). The names of counterparts (in the case of different naming conventions, we use the primary name given by the NED, when available, and otherwise the primary name given by the SIMBAD), the positions of the ADF-S sources and the corresponding nearest counterparts, the angular distances of counterparts from the ADF-S sources, as well as their redshifts, when available, are given in the Table A.1, available from the database of CDS. In this table, we present: the ADF-S identification number, the name of the nearest identified counterpart, right ascension and declination of both ADF-S source and the counterpart, the angular distance between them, and the redshift of the counterpart, when available. Flux densities of ADF-S sources and their identified counterparts, in four FIR bands, are given in CDS Table A.2. The flux densities of counterparts in all the other wavebands, always in the units of Janskys, are given in CDS Tables A.3-A.8. 

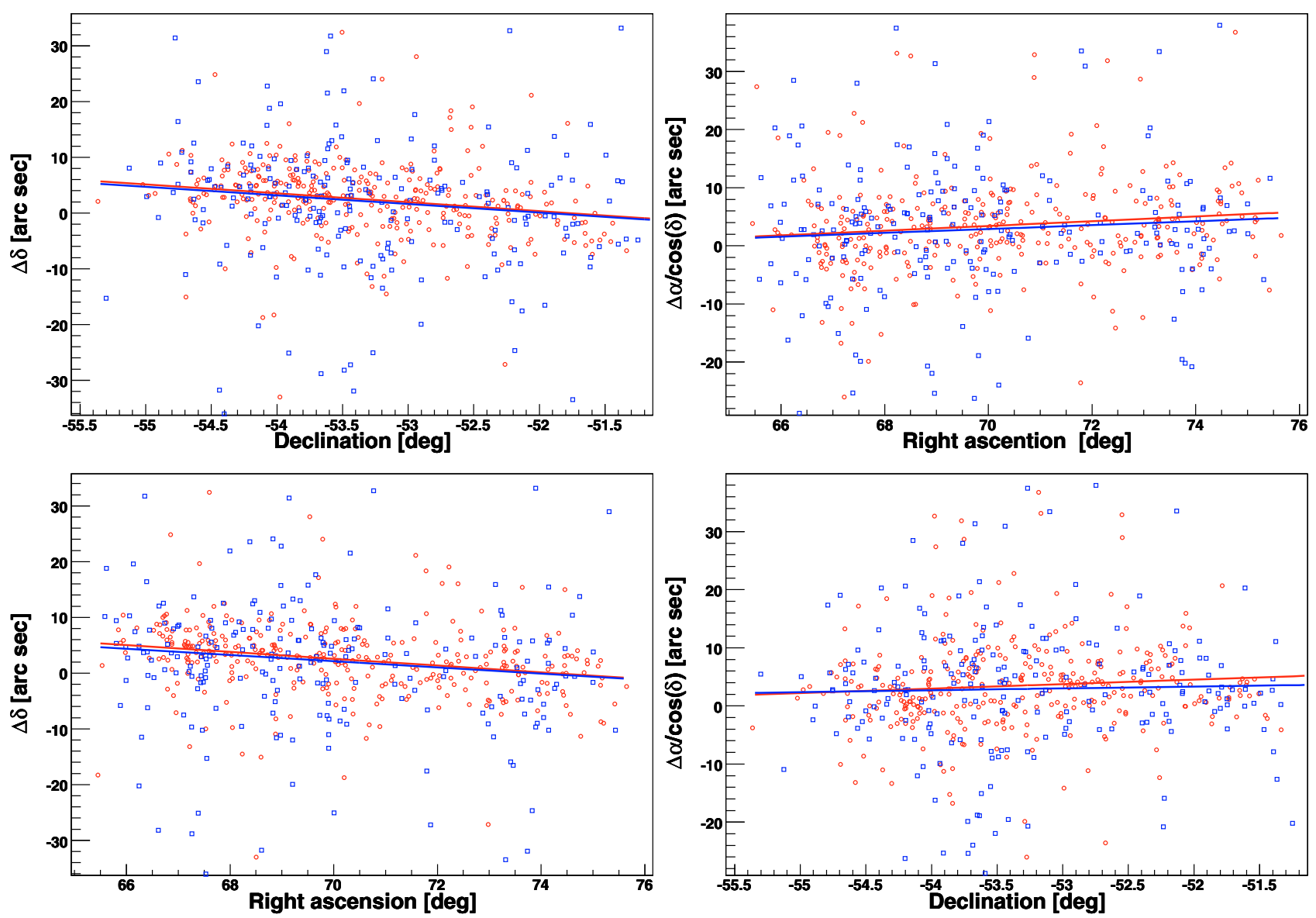

Fig. 3. Dependence of the deviation of the position of counterparts of the ADF-S sources in right ascension and declination, as a function of right ascension and declination. As in Fig. 2, open circles correspond to sources from the $10 \sigma$ catalog and open squares - to sources from the faint part of the $6 \sigma$ catalog. The solid lines correspond to the best rms fits for the bright and faint samples.

Table 1. Best rms fit of the positional deviation.

\begin{tabular}{lcc}
\hline \hline Deviation & $10 \sigma$ catalog & $6 \sigma$ catalog \\
\hline$\Delta \alpha / \cos (\delta)$ vs $\alpha$ & slope & slope \\
$\Delta \delta$ vs $\delta$ & $-1.6 \pm 0.5$ & $+0.3 \pm 0.2$ \\
$\Delta \delta$ vs $\alpha$ & $-0.6 \pm 0.2$ & $-1.6 \pm 0.5$ \\
$\Delta \alpha / \cos (\delta)$ vs. $\delta$ & $+0.8 \pm 0.5$ & $+0.3 \pm 0.2$ \\
\hline & amplitude & amplitude \\
$\Delta \alpha / \cos (\delta)$ vs. $\alpha$ & $-25 \pm 12$ & $-20 \pm 12$ \\
$\Delta \delta$ vs. $\delta$ & $-83 \pm 24$ & $-80 \pm 24$ \\
$\Delta \delta$ vs. $\alpha$ & $+45 \pm 10$ & $+41 \pm 11$ \\
$\Delta \alpha / \cos (\delta)$ vs. $\delta$ & $+45 \pm 28$ & $+20 \pm 26$ \\
\hline
\end{tabular}

As shown in Fig. 4, the sources with multiple counterparts appear mainly in a particular part of the ADF-S, where the nearby cluster of galaxies Abell S0463 is located. We also observe that in the region corresponding to this cluster, there are very few unidentified sources. This may be partially related to the higher local density of galaxies in this part of the field, which increases the risk of a chance coincidence of the angular position of the ADF-S source with one of the cluster galaxies. On the other hand, however, this cluster has been intensely observed in the past, and the optically bright galaxies in this area are sampled much more clearly and deeper than in the remainder of the ADF-S. If we assume that most of the identifications in the
Table 2. Classification of identified ADF-S sources.

\begin{tabular}{llcc}
\hline \hline Catalogs: & & $10 \sigma$ & $6 \sigma$ \\
\hline Galaxies & & 314 & 518 \\
& infrared galaxies & 144 & 176 \\
& radio-loud galaxies & 12 & 13 \\
& galaxies in a cluster of galaxies & 33 & 40 \\
& galaxies in a pair of galaxies & 2 & 2 \\
& interacting galaxies & 2 & 2 \\
& low surface brightness galaxies & 1 & 1 \\
& Seyfert-1 galaxies & 1 & 2 \\
Stars & starburst galaxies & 1 & 1 \\
IR sources & & 5 & 6 \\
Radio sources & & 3 & 3 \\
Quasars & & 6 & 13 \\
X-ray sources & & 1 & 2 \\
& & - & 1 \\
\hline
\end{tabular}

cluster area are correct, we can then deduce that the unidentified objects in the other part of the field are nearby galaxies, which have not been observed before because of their relatively low surface brightness.

\subsection{Number counts}

A similar conclusion that the bright ADF-S sources are mostly nearby galaxies can be deduced by analyzing number counts 


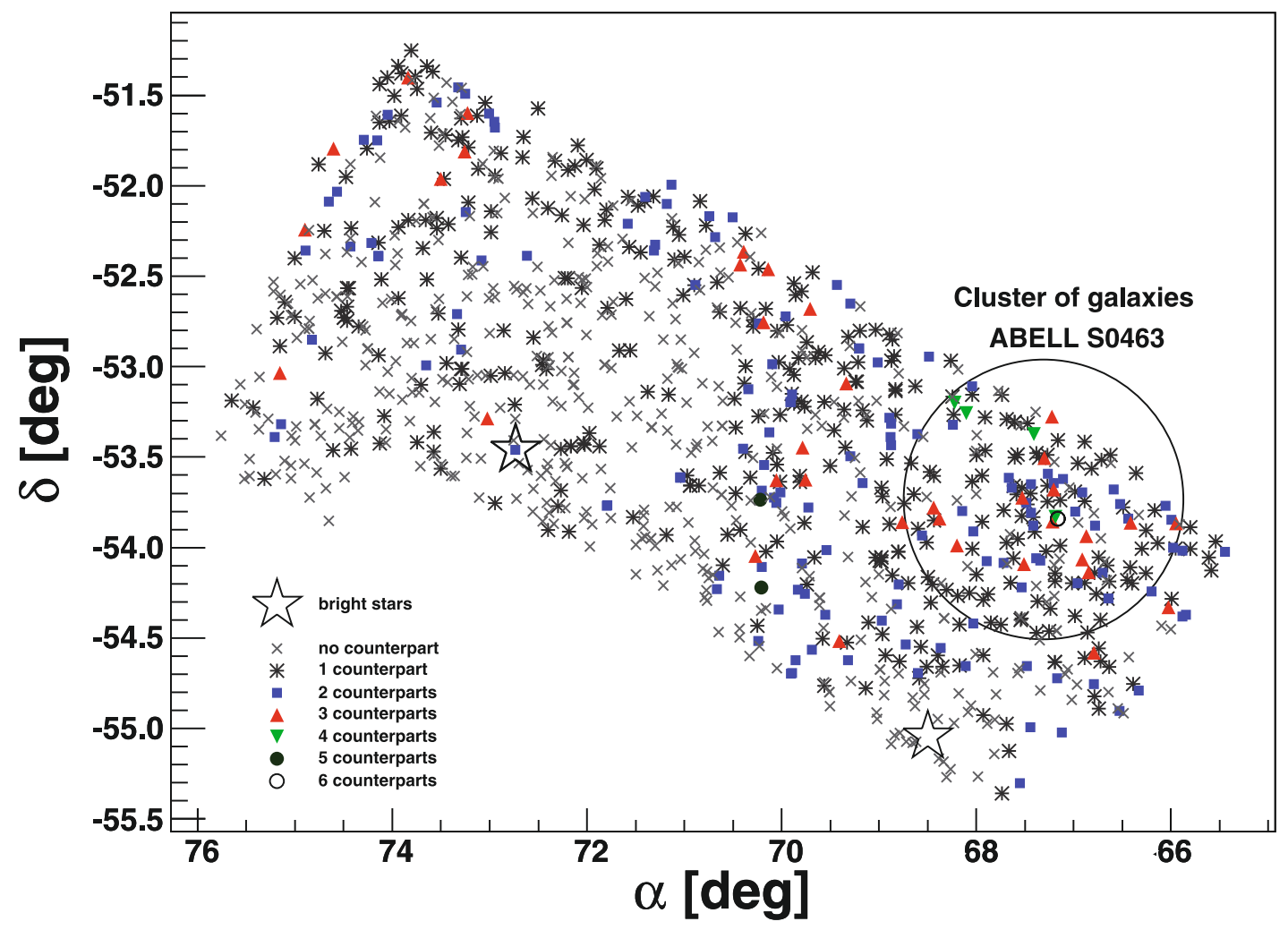

Fig. 4. The map of the ADF-S with the positions of the identified and unidentified objects marked. Sources for which no identification was found (455 sources) are marked as $\times$-s. Sources with one counterpart (362) are indicated by small stars, sources with two counterparts (139) are marked as full squares, those with three counterparts (36) - as triangles, with four counterparts (4) - as upside-down triangles, with five counterparts (2) - as full circles and with six counterparts (2) - as open circles. The positions of two optically bright stars (around 3 and 6 mag) present in the ADF-S are indicated by large, open stars. One of these stars is a double system and an X-ray source; we have also identified it as one of the ADF-S sources. The position of the cluster of galaxies Abell S0463 at $z \sim 0.04$ is indicated by a large, open circle.

of our sample, which is presented in Fig. 5 (for a more detailed analysis of the number counts of the ADF-S galaxies, see Shirahata et al., in prep.). In a homogeneous Euclidean Universe, it is expected that the cumulative number counts of sources follow a power law $N(>S) \propto S^{-3 / 2}$ (for cosmological discussions, see, e.g., Peebles 1993). This relation does not depend on the luminosity function of the sources. The number counts of nearby galaxies can usually be fitted well by a power law with a slope -1.5 . This relation deviates from the Euclidean slope when the cosmological and evolutionary effects become important (e.g., Yoshii \& Takahara 1988; Metcalfe et al. 1996). Thus, the deviation of the measured number counts of the sample from the Euclidean slope at bright flux densities may be a simple test of the completeness of the sample to first approximation.

As shown in Fig. 5, the number counts for all the FIR-bright ADF-S sample, from the $90 \mu \mathrm{m}$ WIDE-S measurement, are quite well reproduced by the Euclidean number counts, which are only slightly lees steep. This implies that these objects are mainly nearby galaxies. The number counts of identified sources are less steep, while the number counts of unidentified sources are steeper than Euclidean. Together with the above discussion, this, implies that the unidentified sources are a less luminous (and, therefore, more difficult to observe optically) nearby population of galaxies. As expected, galaxies with known redshifts represent the brightest sample with the flattest number counts.

For the twice larger $6 \sigma$ sample, the situation changes only slightly. The comparison of slopes of the best rms fits to the observed number counts for both samples are given in Table 3 .

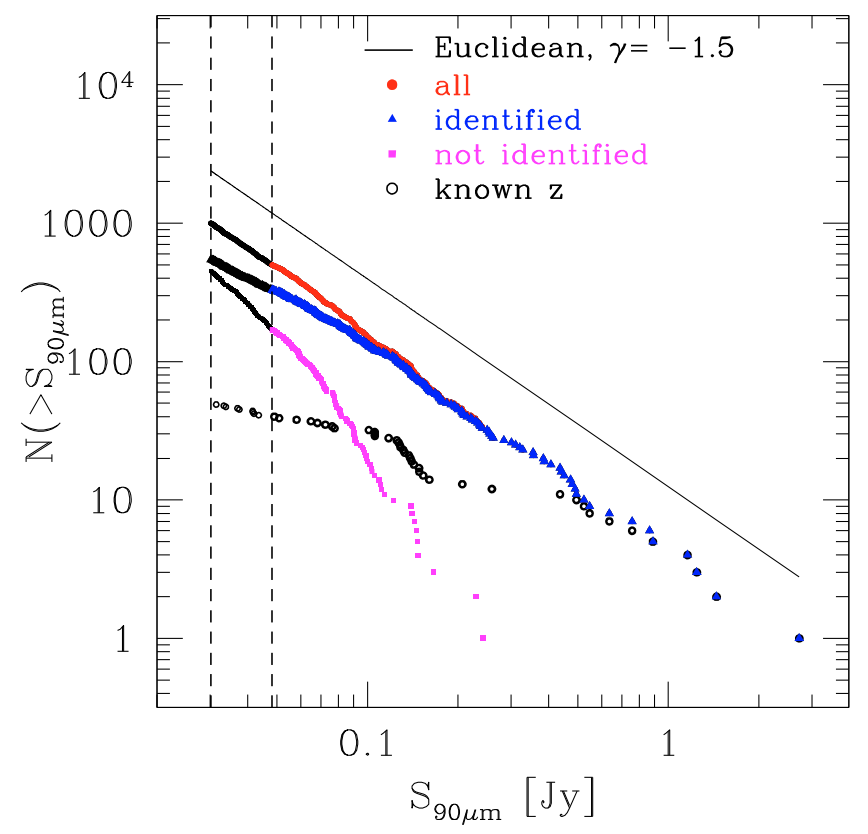

Fig. 5. Integral number counts of objects in the the $10 \sigma$ and $6 \sigma$ ADF-S samples in $90 \mu \mathrm{m}$. Filled circles correspond to all the 6 and $10 \sigma$ datasets, triangles - to the identified objects, squares - to non-identified objects and open circles - to objects with known $z$. The dashed vertical lines mark the limiting luminosities of $10 \sigma$ and $6 \sigma$ catalogs. For comparison, the ideal Euclidean case, with an arbitrary amplitude, has been shown as a solid line. 
Table 3. Slope of number counts of ADF-S sources.

\begin{tabular}{lcc}
\hline \hline Objects & $10 \sigma$ sample & $6 \sigma$ sample \\
& $S_{90 \mu \mathrm{m}}>0.0482 \mathrm{Jy}$ & $S_{90 \mu \mathrm{m}}>0.0301 \mathrm{Jy}$ \\
\hline All & $-1.63 \pm 0.03$ & $-1.58 \pm 0.02$ \\
Identified & $-1.45 \pm 0.03$ & $-1.34 \pm 0.02$ \\
Not identified & $-3.15 \pm 0.09$ & $-2.55 \pm 0.04$ \\
Known $z$ & $-0.84 \pm 0.05$ & $-0.77 \pm 0.04$ \\
\hline
\end{tabular}

In total, we conclude that the bright part of the FIR-selected sample of celestial objects in the ADF-S consists mainly of nearby galaxies. The average properties of these galaxies are examined in the following.

\subsection{Redshift distribution}

The redshift information is available for 48 galaxies from the full $6 \sigma$ sample. The only two high redshift sources are quasars, VV2006 J044011.9-524818, located at $z=1.053$ and HE 04355304 , located at $z=1.232$, and two more galaxies are located at $z \sim 0.25$. All the other sources are nearby galaxies at $z<$ 0.1. The redshift distribution of these galaxies, shown in Fig. 6, demonstrates that a large fraction of them belongs to a cluster Abell S0463 at $z \sim 0.04$.

\subsection{Completeness of identifications}

In Fig. 7, we present the ratio of identified to non-identified sources, as well as that of objects with measured redshifts to the total number of sources in subsamples of different limiting flux density at $90 \mu \mathrm{m}$. This shows that the sample of identified sources is $100 \%$ complete to $S_{90}=0.25 \mathrm{Jy}$, which corresponds to the first 32 sources, and remains more than $90 \%$ complete to $S_{90}=0.105 \mathrm{Jy}$, corresponding to the first 140 sources. For sources fainter than $0.105 \mathrm{Jy}$, the completeness of the catalog begins to decline rapidly to $66 \%$ for the $10 \sigma$ and $55 \%$ for the complete $6 \sigma$ sample.

This incompleteness has to be taken into account if we wish to apply the conclusions of the analysis of the identified sample to the entire FIR-bright ADF-S dataset.

\subsection{Galaxy morphologies and environment}

Among the identified galaxies, 67 in the $10 \sigma$ sample and 10 in the fainter part of the $6 \sigma$ sample have determined morphologies. Most of them (but not all) belong to the cluster Abell S0463 and were identified by Dressler (1980a). The redshift of the cluster is $z \sim 0.0394$ (Abell et al. 1989). It is a regular (type I-II in the Bautz-Morgan classification), moderately rich (population 84), lenticular-rich galaxy cluster and was used as a typical regular cluster in a sample investigated by Dressler (1980b). We can then assume that our morphological sample is - in large part - representative of the nearby bright galaxy population. However, a rich cluster may introduce some bias towards dense environments.

In Table 4, we present the statistics of the galaxy types in our sample compared to the frequencies of different types usually found in an optically bright galaxy population in the nearby Universe (de Vaucouleurs 1963). The first almost expected but remarkable observation is that of a high percentage of peculiar galaxies in our sample. The high FIR luminosity of dust in these objects reflects the ongoing star forming processes induced in them by interactions with other galaxies. We observe slightly more spiral galaxies than expected in an optically-selected

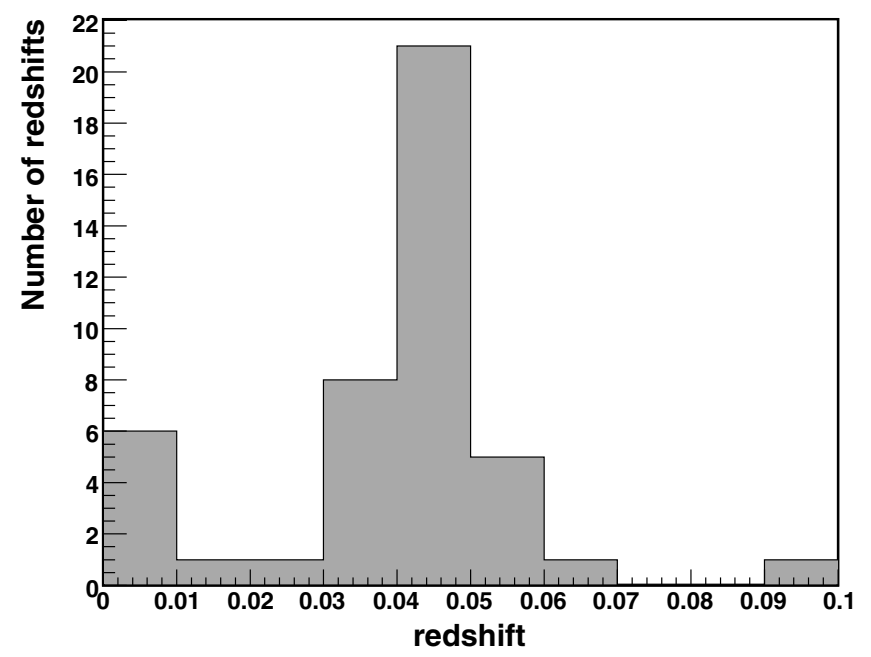

Fig. 6. The redshift histogram of 44 counterparts of the ADF-S objects with known redshifts in 0.01 bins. Four objects with redshifts higher than 0.2 are not shown here. These are: one galaxy at $z=0.2591$, one Seyfert- 1 galaxy at $z=0.243$, and two quasars: HE 0435-5304, located at $z=1.232$, and VV2006 J044011.9-524818, located at $z=1.053$.

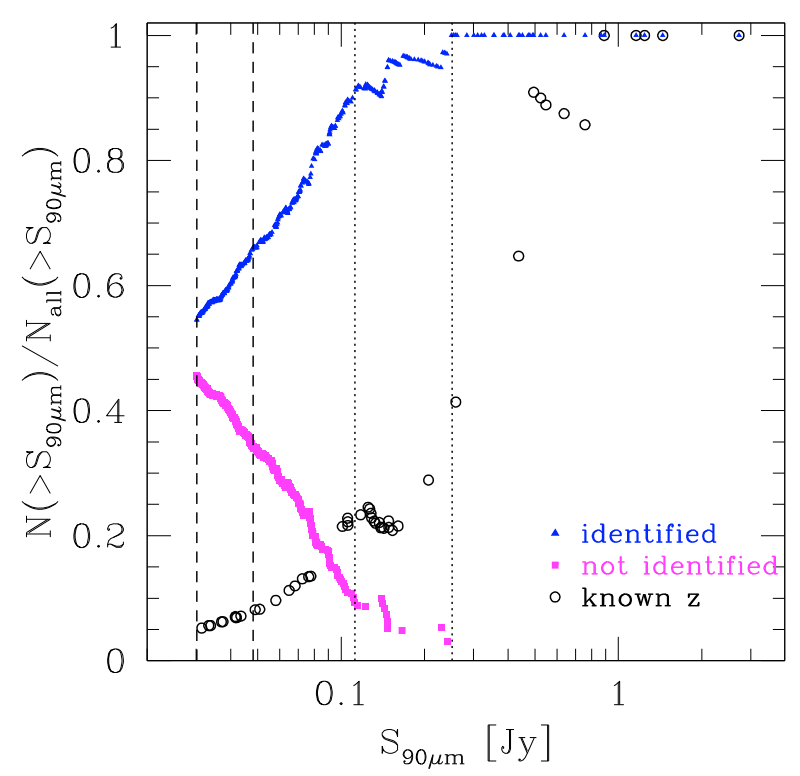

Fig. 7. Ratios of identified to unidentified sources, and of sources with known redshift, with respect to the total number of sources in the subsamples of a different $90 \mu \mathrm{m}$ limiting luminosity. As in Fig. 5, triangles correspond to the identified objects, squares - to non-identified objects and open circles - to objects with known $z$. The dashed vertical lines mark the limiting luminosities of $10 \sigma$ and $6 \sigma$ catalogs. The dotted vertical lines mark the limits of $100 \%$ and $90 \%$ completeness of the catalog of counterparts.

sample but - given the number statistics - this overrepresentation of spirals is insignificant. This is compensated by a significantly (even given the small number statistics) lower amount of elliptical galaxies in the sample. Moreover, among the five elliptical galaxies identified one belongs to a pair of interacting galaxies and one is a Seyfert-1 active galaxy. These peculiarities probably explain the unusual FIR luminosity of these two galaxies and cause the fraction of seemingly normal elliptical galaxies in our sample to be even lower. 
Table 4. 77 ADF-S galaxies with known morphological types.

\begin{tabular}{lrrrrc}
\hline \hline \multicolumn{5}{c}{$10 \sigma$ catalog } & \multicolumn{2}{c}{$6 \sigma$ catalog } & \\
\hline & Number & \multicolumn{1}{c}{ Frequency } & Number & Frequency & $\begin{array}{c}\text { Usual frequency } \\
\text { among bright galaxies }\end{array}$ \\
\hline spiral & 45 & $67 \% \pm 10 \%$ & 50 & $65 \% \pm 9 \%$ & $61 \%$ \\
lenticular & 15 & $22.5 \% \pm 6 \%$ & 17 & $22 \% \pm 5 \%$ & $22 \%$ \\
elliptical & 4 & $6 \% \pm 3 \%$ & 5 & $6.5 \% \pm 3 \%$ & $13 \%$ \\
irregular & 1 & $1.5 \% \pm 1.5 \%$ & 3 & $4 \% \pm 2 \%$ & $3.5 \%$ \\
compact & 2 & $3 \% \pm 2 \%$ & 2 & $2.5 \% \pm 2 \%$ & - \\
peculiar & 8 & $12 \% \pm 4 \%$ & 8 & $10 \% \pm 4 \%$ & $1 \%$ \\
\hline
\end{tabular}

Notes. ${ }^{(a)}$ As in de Vaucouleurs (1963) .

The fraction of lenticular (S0) galaxies in our sample was found to be practically identical to that of normal optically bright galaxies. The issue of dust in lenticular galaxies has been widely discussed. Lanes of dust and gas have been found in many objects of this type (e.g., Danks et al. 1979; Sil' chenko \& Afanasiev 2004). IRAS observations found that $\sim 68 \%$ of lenticular galaxies, compared to $\sim 45 \%$ of ellipticals, contain cool dust (Knapp et al. 1989) and remain visible in the infrared. Our results are consistent with the conclusion of Roberts \& Haynes (1994) that FIR detection provides the most prominent distinction between ellipticals and spirals, lenticulars in this context remaining an intermediate (and slightly elusive) type. Spitzer observations of three lenticular galaxies found that even if their bulge-to-disk ratios support their classification as lenticulars, they contain warm dust that forms structure similar to spiral arms (Pahre et al. 2004). That the fraction of lenticular galaxies in the FIR selected sample is the same as in the optically selected sample of bright galaxies is additional evidence that warm dust in lenticular galaxies may be common.

Although lenticulars were originally introduced as a transition class between elliptical and spiral galaxies (Hubble 1936), there is mounting evidence that they are more complex objects. It has been suggested (van den Bergh 1994) that lenticulars can be divided into two subpopulations that have different formation histories. Some faint lenticulars could be produced by secular formation processes at early epochs or by the slow stripping of gas from spirals in the cluster environment (Abadi et al. 1999). The more luminous ones could instead be produced by the mergers of spiral galaxies (Bekki 1998). Other processes, such as gas starvation or gas ejection by active nuclei, should probably also be taken into account when describing the evolution of lenticular galaxies (van den Bergh 2009). This more complex scenarios seem to be supported by observations of lenticulars, e.g., in the near infrared (Barway et al. 2009). Detection of a significant population of lenticular galaxies in the FIR proves that substantial amount of cold dust is quite normal in this class of galaxies and is additional evidence of their complex formation process.

\subsection{Correlation function}

A careful analysis of the correlation function and clustering properties of galaxies in the ADF-S data will be given in Kawada et al. (in prep.). Here we present a simple analysis of clustering of galaxies from our sample, to examine the quality of the data and any possible biases.

The correlation function is the simplest statistical measurement of clustering, as a function of scale (angular or spatial).
It corresponds to the second moment of the galaxy distribution. To compute the angular correlation function $\omega$ of the ADF-S galaxies as a function of the angular scale $\theta$, we adopted the Landy-Szalay estimator (Landy \& Szalay 1993), which expresses $\omega(\theta)$ as

$\omega(\theta)=\frac{N_{R}\left(N_{R}-1\right)}{N_{G}\left(N_{G}-1\right)} \frac{G G(\theta)}{R R(\theta)}-\frac{N_{R}-1}{N_{G}} \frac{G R(\theta)}{R R(\theta)}+1$.

In this equation, $N_{G}$ and $N_{R}$ are the mean densities (or, equivalently, the total numbers) of objects, respectively, in the galaxy sample and a catalog of random points. The random points are distributed within the same survey area and with the same angular selection biases as galaxies in the ADF-S catalog. We denote by $G G(\theta)$ the number of independent galaxy-galaxy pairs with separations of $\theta$ to $\theta+\mathrm{d} \theta$, by $R R(\theta)$ - the number of independent random-random pairs within the same interval of separation, and by $G R(\theta)$ - the number of galaxy-random pairs.

In the nearby Universe, the angular correlation function of galaxies can usually be fitted by a power law $\omega(\theta)=A_{\omega} \theta^{1-\gamma}$, where an amplitude $A_{\omega}$ is a measure of clustering strength and $\gamma$ infers its scale dependence. In practice, because of the finite size of the survey, the measured $\omega(\theta)$ is a biased estimator of the true correlation function and becomes underestimated on the large scale. The correction factor that needs to be applied is related to an integral-constrained IC, (Peebles \& Groth 1976),

$\mathrm{IC}=\frac{1}{\Omega^{2}} \iint \omega(\theta) \mathrm{d} \Omega_{1} \mathrm{~d} \Omega_{2}$,

where $\Omega$ is the area of the observed field. The measured correlation function can then be written as

$\omega(\theta)=A_{\omega}\left(\theta^{1-\gamma}-\mathrm{IC}\right)$.

Correlation functions measured for the $10 \sigma$ and $6 \sigma$ ADF-S samples are shown in Fig. 9, were we plot the best-fit power law with the IC applied. In this case, we do not use the covariance matrix to correct for the correlation between the bins, since the precise measurement of the clustering parameters is not our objective. For the same reason, the error bars are simple Poissonian errors. Thus, both the fits and the errors in our calculation are no more than indicative. However, they provide sufficient information to compare the properties of different subsets of our data. The clustering amplitude for the full $10 \sigma$ ADF-S $90 \mu \mathrm{m}$ sample is equal to $1.1 \times 10^{-3}$ with the slope $\gamma=2$, which is not very different from that found for other infrared galaxy surveys, for example SWIRE (Oliver et al. 2004; de la Torre et al. 2007). In case of the $6 \sigma$ catalog, we observe a lower clustering amplitude for the 


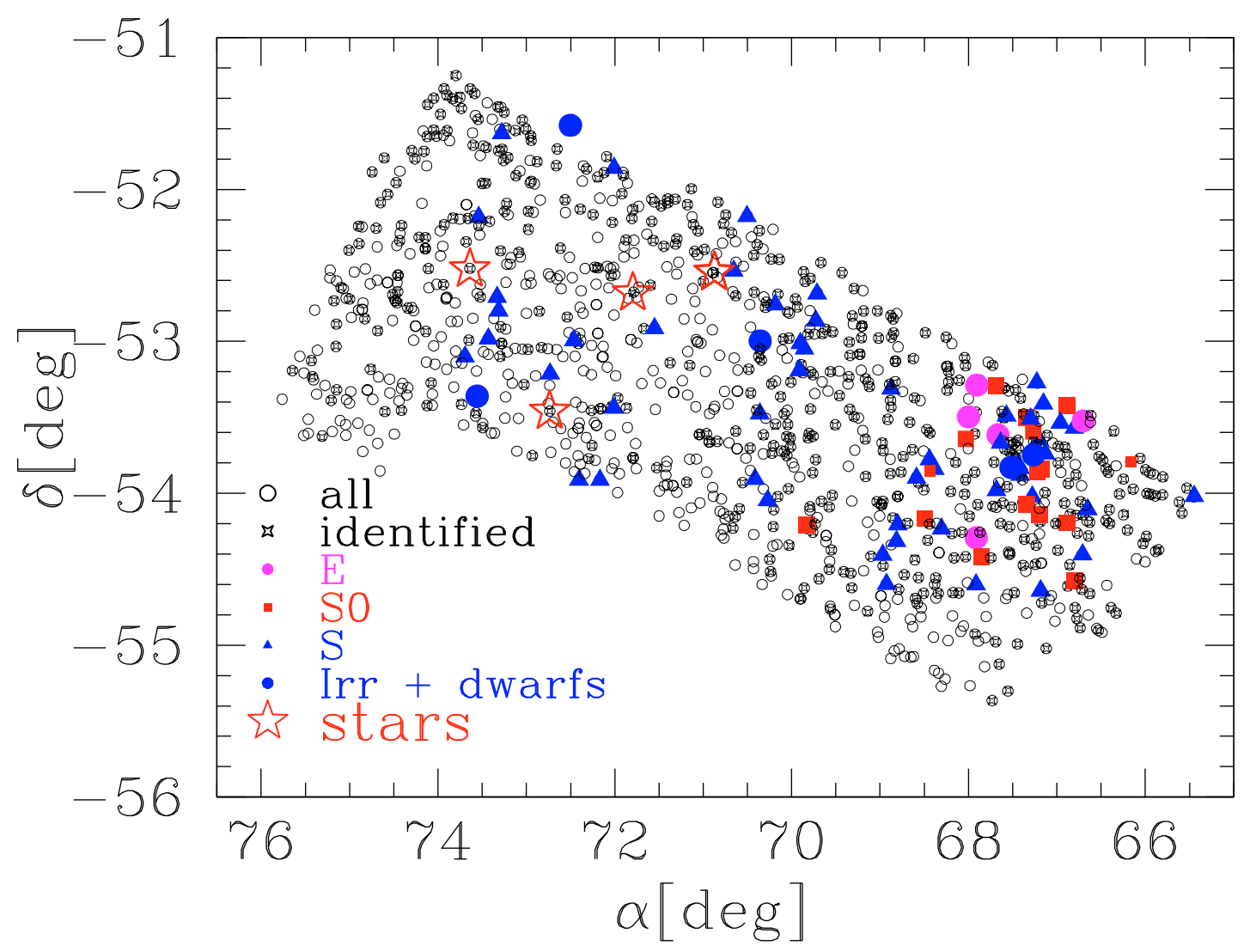

Fig. 8. Positions of galaxies with known morphological types in the ADF-S. In this plot, positions of all the ADF-S sources are marked by open circles. Identified sources are shown as small stars. The elliptical galaxies are shown as full circles, lenticular galaxies as full squares, spiral galaxies as full triangles, and irregular or dwarf galaxies as full circles. Positions of identified stars are shown with large, open stars; however, these identifications are most probably the effect of contamination. We can observe that all the elliptical and lenticular galaxies are located in the region of the galaxy cluster Abell S0463 at $z \sim 0.04$. Their morphologies were mostly determined by Dressler (1980a). The spiral and irregular galaxies, however, are distributed throughout the field.
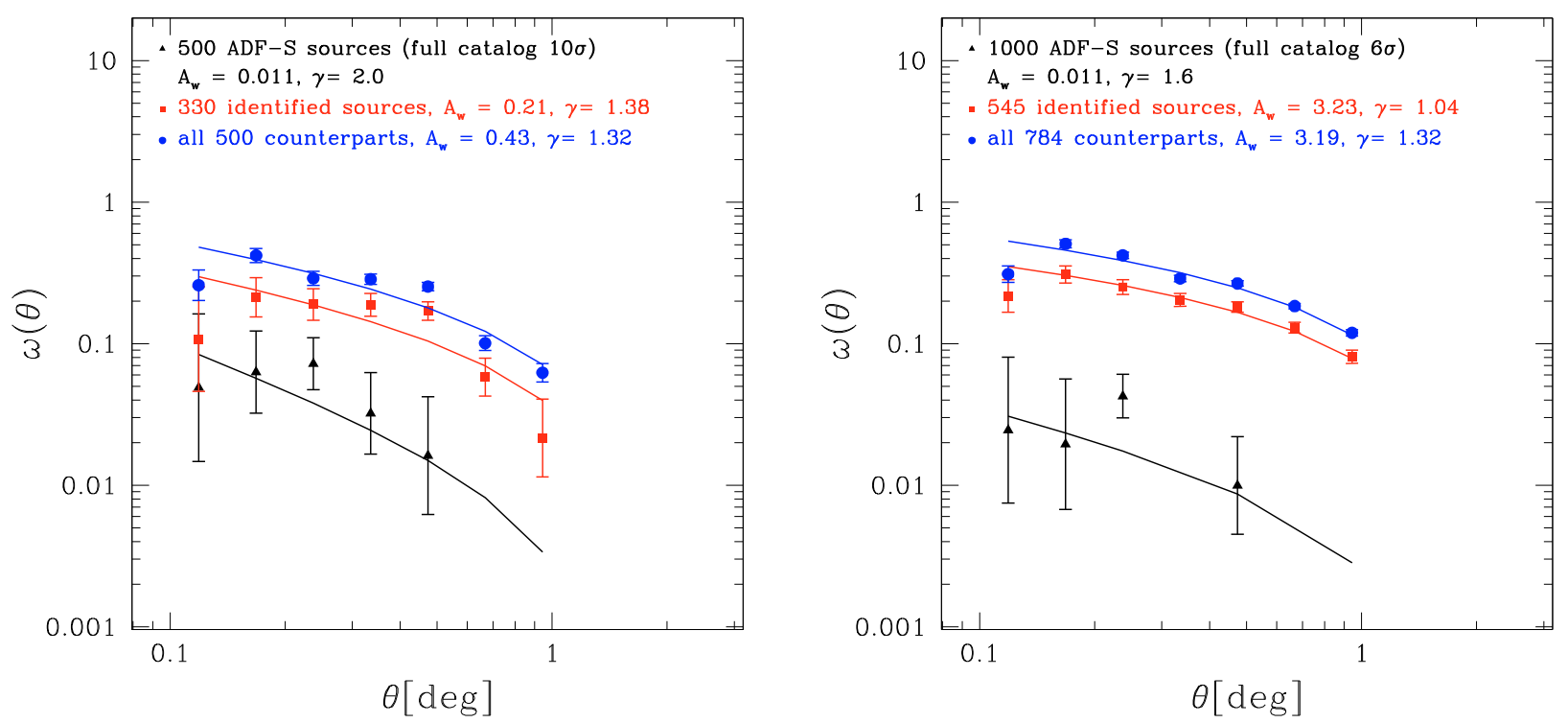

Fig. 9. Correlation function of the $10 \sigma$ (left panel) and $6 \sigma$ (right panel) ADF-S samples. Triangles correspond to the full $10 \sigma$ and $6 \sigma$ dataset of ADF-S sources, squares to the samples of the most nearby counterparts of identified sources, and circles to samples constructed from all the counterparts of the identified ADF-S sources at the angular distance smaller than 40" from the ADF-S source. The corresponding best-fit power law with an integral constraint $\omega(\theta)=A\left(\theta^{-\gamma}-\right.$ IC) is shown in each case. We observe a lower clustering of the full sample and a higher clustering of the identified sample, compared to the $10 \sigma$ catalog. Correlation function of all counterparts found shows in both cases a deficit of pairs on small scales, which suggests that the level of source confusion is higher than the ratio of secondary counterparts, i.e., $34 \%$ of the sample. 
whole sample, which may be attributed to us dealing with fainter and hence less massive sources. When computing the correlation function for only identified sources, we have in both cases a strong clustering signal. The correlation function becomes less steep and the clustering amplitude much higher. This confirms that the identified sources on the sky belong to the brighter local population of galaxies, which resides in denser environments.

In the case of the $6 \sigma$ sample, the clustering amplitude of identified sources is even higher than for the brighter $10 \sigma$ sample. This increase in the clustering amplitude probably reflects the identification process, more for fainter sources than brighter ones, being biased by the cluster of galaxies Abell S0463 in the ADF-S. The objects in the region occupied by this cluster have been closely studied, and hence are represented more in the public databases. Thus, we should be aware that all conclusions drawn from the catalog of counterparts of $6 \sigma$ sources are biased towards dense environments, compared to the $10 \sigma$ catalog. The high clustering amplitude of the counterparts to the $10 \sigma$ sources should probably be partially attributed to this bias, even if the effect is clearly smaller. Deriving real-space clustering parameters requires more detailed estimation of the redshift distribution of our sources, as well as the proper treatment of the possible biases, and will be the subject of future studies.

When analyzing $\omega(\theta)$, a lack of pairs is clearly evident on scales smaller than $\sim 0.2 \mathrm{deg}$. This is common to all measurements with IR data and is caused mainly by source confusion. The scale where the deficit of pairs occurs in the case of the closest counterparts is, as expected, the same as for the full dataset. This observation assures us that it is really caused by source confusion and not, for instance, by incompleteness in the point source identification process.

To examine the possible impact of source confusion on our data, we experimented by assuming that all counterparts found in the $40^{\prime \prime}$ range, not only the nearest ones, can contribute to the FIR flux and, therefore, to the clustering signal. As expected, the clustering amplitude of a sample constructed in this way is even higher. The deficit of pairs on small scales decreases significantly but remains. This suggests that the number of sources missing because of source confusion in our sample is even greater than the number of identified secondary counterparts, i.e., $34 \%$ of the sample.

More careful examination of the data is required to determine the percentage of secondary counterparts that contributes to the IR flux of ADF-S sources. At the same time, as mentioned above, even the assumption that all the identified secondary counterparts could contribute to the clustering signal does not ensure a sufficiently large number of close galaxy pairs in our data to obtain a power-law shape of the correlation function. It is then possible that some other unresolved (and non-identified) extragalactic IR sources contribute to the flux of the identified sources. In addition, since many IR-bright objects in our sample are peculiar or interacting, we may suspect that even in a greater number of cases not detected interacting partners do exist and might contribute to the IR flux. The conclusion is that the smallscale environment of the galaxies observed in the infrared should be studied in the future even more carefully.

\section{Spectral energy distributions}

As mentioned in Introduction, SEDs provide important clues to the origin of the source radiation. The deep images in the AKARI filter bands provide the opportunity to analyze SEDs and update models of their interstellar dust emission.

\subsection{SED models}

From the $10 \sigma$ sample, we selected 47 galaxies with highest quality photometry available to fit the models of their SEDs. The results are presented in Figs. 13-18. The main selection criterion was to have at least three data points in the infrared range of the spectrum, to ensure that the dust properties could be modeled reliably. In the selected sample, 23 galaxies have had their morphologies determined. There is one elliptical galaxy, two compact galaxies (one of them which is a starburst blue compact dwarf), five lenticular galaxies, and 15 spiral galaxies.

To fit the SEDs of these galaxies, we use three methods. We first applied a modified black body to the dust-emission-affected part of the galaxy SED, and a black body to the stellar emission part. The results are presented in Sect. 4.1.1. Since the galaxies in our sample are often evolved, a single black body often provides a poor fit to the observed SEDs for the stellar emission part of the SED. In future work, we plan to apply more sophisticated stellar population synthesis models with more realistic star formation history to model the stellar component of the SED.

It is widely known that some dust components in galaxies cannot establish an equilibrium with ambient radiation field. These components produce strong mid-IR (MIR) emission which extends across a wide range of wavelengths and cannot be reproduced well by a modified black body (e.g., Purcell 1976; Draine \& Anderson 1985; Li \& Draine 2001; Draine \& Li 2001; Takeuchi et al. 2003, 2005c). To deal with this MIR emission, we should use more sophisticated models in our fitting. In addition to the modified black-body model, we then used the models of Dale \& Helou (2002) and Li \& Draine (2001). These more refined models in most cases succeeded in reproducing the MIR part of the dust emission. The results are presented Sects. 4.1.2 and 4.1.3.

To galaxy SEDs, we used all available measurements from the ADF-S catalog, which are listed in Table A.2 available from the database of CDS and data from public databases (listed in the Tables A.3-A.6, also available from the CDS), only excluding several of the most unreliable measurements, as discussed below.

To fit Dale \& Helou (2002) and Li \& Draine (2001) models, we used all four bands from the ADF-S measurements, when available, i.e., N60 $(65 \mu \mathrm{m})$, WIDE-S $(90 \mu \mathrm{m})$, WIDE-L $(140 \mu \mathrm{m})$, and $\mathrm{N} 160(160 \mu \mathrm{m})$, four IRAS bands $(12 \mu \mathrm{m}, 25 \mu \mathrm{m}$, $60 \mu \mathrm{m}$, and $100 \mu \mathrm{m})^{3}$, in one case of a galaxy NGC 1705 seven Spitzer bands $(3.6 \mu \mathrm{m}, 4.5 \mu \mathrm{m}, 5.8 \mu \mathrm{m}, 8 \mu \mathrm{m}, 24 \mu \mathrm{m}, 70 \mu \mathrm{m}$, and $160 \mu \mathrm{m})$, and in one case of a galaxy ESO 157-49 one ISOPHOT band $(170 \mu \mathrm{m})$. We included the uncertainties in all these data points in the fitting process. Data from the ADF-S were not treated in any special way. To determine the best-fit models, we used a $\chi^{2}$ test, the details of which in the case of simple black-body and dust models are explained in the corresponding sections below.

Among the 47 considered galaxies, there are 15 objects with known redshifts. The SEDs of these 15 galaxies are fitted and presented in the rest frame. In contrast, the SEDs of the remaining objects are fitted and presented in the observed frame.

The parameters determined for all three methods are summarized in Table 5. As complementary information, we summarize the morphological and environmental properties of galaxies used in the fitting in Table 6.

\footnotetext{
${ }^{3}$ Note that in some cases the IRAS catalog provides only the upper limits to the IR flux.
} 
A\&A 514, A11 (2010)

Table 5. Parameters of the fitted models of SEDs of 47 ADF-S galaxies with the highest quality photometric data.

\begin{tabular}{|c|c|c|c|c|c|c|c|}
\hline \multirow{2}{*}{$\begin{array}{c}\text { ID } \\
(\mathrm{ADF}-\mathrm{S})\end{array}$} & \multicolumn{2}{|c|}{ Modified black body } & \multirow{2}{*}{$\begin{array}{c}\text { Dale \& Helou } \\
\alpha\end{array}$} & \multicolumn{4}{|c|}{ Li \& Draine } \\
\hline & Dust temp. $[\mathrm{K}]$ & Stellar temp. $[\mathrm{K}]$ & & Umin & Umax & PAH $(\%)$ & Name \\
\hline 1 & $26.9 \pm 0.2$ & $2800 \pm 40$ & 2.4375 & 4.00 & 4.00 & 2.37 & LMC2 \\
\hline 2 & $37.8 \pm 0.3$ & $3060 \pm 30$ & 2.1250 & 0.10 & 100 & 4.58 & MW3.1 \\
\hline 3 & $22.9 \pm 0.2$ & $2950 \pm 30$ & 2.1250 & 0.50 & 0.50 & 2.37 & LMC2 \\
\hline 4 & $36.3 \pm 0.1$ & $7000 \pm 120$ & 1.0000 & 1.20 & 100 & 2.37 & LMC2 \\
\hline 5 & $33.6 \pm 0.3$ & $3260 \pm 50$ & 1.3125 & 0.10 & 100 & 2.37 & LMC2 \\
\hline 6 & $32.6 \pm 0.3$ & $2110 \pm 70$ & 1.3125 & 5.00 & 5.00 & 2.37 & LMC2 \\
\hline 7 & $47.8 \pm 0.4$ & $5110 \pm 50$ & 0.0625 & 8.00 & $1 \mathrm{e} 4$ & 2.37 & LMC2 \\
\hline 8 & $36.8 \pm 0.4$ & $2020 \pm 70$ & 0.0625 & 0.10 & $1 \mathrm{e} 4$ & 1.12 & MW3.1 \\
\hline 9 & - & $3890 \pm 50$ & 1.1875 & 0.80 & $1 \mathrm{e} 4$ & 3.90 & MW3.1 \\
\hline 10 & $27.8 \pm 0.2$ & $3230 \pm 30$ & 4.0000 & 2.00 & 2.00 & 2.37 & LMC2 \\
\hline 11 & $28.9 \pm 0.3$ & $3030 \pm 40$ & 4.0000 & 2.00 & 2.00 & 2.37 & LMC2 \\
\hline 12 & $36.0 \pm 0.6$ & $2070 \pm 120$ & 4.0000 & 5.00 & $1 \mathrm{e} 4$ & 2.37 & LMC2 \\
\hline 13 & $33.2 \pm 0.4$ & - & 4.0000 & 0.10 & 100 & 0.75 & LMC2 \\
\hline 14 & $32.4 \pm 0.4$ & - & 0.0625 & 8.00 & 100 & 2.37 & LMC2 \\
\hline 15 & $32.1 \pm 0.2$ & $1980 \pm 10$ & 0.0625 & 0.10 & 100 & 0.75 & LMC2 \\
\hline 16 & $28.2 \pm 0.7$ & - & 0.0625 & 0.10 & 100 & 4.58 & MW3.1 \\
\hline 17 & $33.2 \pm 0.6$ & $5890 \pm 60$ & 0.0625 & 0.10 & $1 \mathrm{e} 4$ & 0.75 & LMC2 \\
\hline 18 & $28.8 \pm 0.2$ & $2260 \pm 70$ & 0.0625 & 4.00 & 4.00 & 2.37 & LMC2 \\
\hline 19 & $33.8 \pm 0.1$ & $2050 \pm 10$ & 0.0625 & 15.0 & 15.0 & 0.75 & LMC2 \\
\hline 20 & $33.0 \pm 0.5$ & $1980 \pm 70$ & 0.0625 & 0.10 & $1 \mathrm{e} 4$ & 0.75 & LMC2 \\
\hline 21 & $29.4 \pm 0.2$ & $2210 \pm 10$ & 0.0625 & 4.00 & 4.00 & 2.37 & LMC2 \\
\hline 22 & $38.0 \pm 0.1$ & $2060 \pm 40$ & 4.0000 & 0.10 & 100 & 0.75 & LMC2 \\
\hline 23 & $28.1 \pm 0.3$ & $2340 \pm 70$ & 4.0000 & 0.10 & 100 & 2.37 & LMC2 \\
\hline 24 & $33.4 \pm 0.6$ & $2140 \pm 10$ & 4.0000 & 0.10 & $1 \mathrm{e} 6$ & 0.75 & LMC2 \\
\hline 26 & $43.5 \pm 0.1$ & - & 0.0625 & 4.00 & $1 \mathrm{e} 4$ & 0.75 & LMC2 \\
\hline 27 & $35.7 \pm 0.3$ & - & 0.0625 & 0.80 & 100 & 2.37 & LMC2 \\
\hline 29 & $27.5 \pm 0.8$ & $2950 \pm 20$ & 0.0625 & 0.10 & 100 & 0.75 & LMC2 \\
\hline 30 & $32.7 \pm 0.7$ & $1900 \pm 10$ & 0.0625 & 0.10 & $1 \mathrm{e} 6$ & 4.58 & MW3.1 \\
\hline 31 & $32.8 \pm 0.3$ & $1970 \pm 120$ & 0.0625 & 8.00 & 8.00 & 2.37 & LMC2 \\
\hline 39 & $47.4 \pm 0.4$ & $3500 \pm 60$ & 0.0625 & 0.20 & $1 \mathrm{e} 6$ & 0.47 & MW3.1 \\
\hline 41 & $32.5 \pm 0.1$ & $1950 \pm 10$ & 0.0625 & 12.0 & 12.0 & 0.75 & LMC2 \\
\hline 42 & - & $2210 \pm 30$ & 0.0625 & 0.10 & $1 \mathrm{e} 5$ & 0.75 & LMC2 \\
\hline 43 & $33.3 \pm 0.7$ & $1970 \pm 10$ & 0.0625 & 0.10 & $1 \mathrm{e} 6$ & 0.75 & LMC2 \\
\hline 45 & $28.5 \pm 0.1$ & $4160 \pm 80$ & 3.8750 & 0.10 & 100 & 0.75 & LMC2 \\
\hline 46 & - & $1650 \pm 20$ & 3.8750 & 0.10 & $1 \mathrm{e} 6$ & 4.58 & MW3.1 \\
\hline 48 & $24.3 \pm 0.1$ & $2010 \pm 10$ & 3.8750 & 1.20 & 1.20 & 2.37 & LMC2 \\
\hline 49 & $26.6 \pm 0.1$ & - & 3.8750 & 0.10 & 100 & 0.75 & LMC2 \\
\hline 50 & $33.6 \pm 0.1$ & $3380 \pm 60$ & 3.8750 & 0.10 & $1 \mathrm{e} 6$ & 1.49 & LMC2 \\
\hline 51 & $29.7 \pm 0.2$ & $3120 \pm 30$ & 2.2500 & 0.10 & 100 & 0.75 & LMC2 \\
\hline 52 & - & $2000 \pm 30$ & 2.2500 & 2.00 & $1 \mathrm{e} 6$ & 2.37 & LMC2 \\
\hline 56 & $34.8 \pm 0.1$ & - & 2.2500 & 0.10 & $1 \mathrm{e} 6$ & 4.58 & MW3.1 \\
\hline 64 & $23.1 \pm 0.1$ & $1940 \pm 10$ & 2.2500 & 0.70 & 0.70 & 2.37 & LMC2 \\
\hline 65 & $28.0 \pm 0.1$ & $4600 \pm 110$ & 4.0000 & 1.50 & 1.50 & 2.37 & LMC2 \\
\hline 69 & $26.4 \pm 0.1$ & $1770 \pm 80$ & 4.0000 & 2.00 & 2.00 & 2.37 & LMC2 \\
\hline 73 & - & - & 4.0000 & 1.50 & $1 \mathrm{e} 6$ & 2.37 & LMC2 \\
\hline 82 & $27.8 \pm 0.1$ & - & 4.0000 & 2.00 & 2.00 & 3.19 & MW3.1 \\
\hline 103 & $20.2 \pm 0.1$ & $2910 \pm 50$ & 4.0000 & 0.10 & 0.10 & 2.37 & LMC2 \\
\hline
\end{tabular}

Notes. The table contains ADF-S identification numbers (first column), indicative temperatures of dust and stellar component resulting from the best-fit modified black-body models, the parameter $\alpha$ of the Dale \& Helou (2001) model, three parameters, and the name of the best-fit Li \& Draine (2001) model.

\subsubsection{Modified blackbody model}

In the simplest approach, we modeled the stellar component of galaxies $\left(v=5 \times 10^{13}-10^{15} \mathrm{~Hz}\right.$, i.e., $\left.\lambda=0.3-6 \mu \mathrm{m}\right)$ with the black-body spectrum

$B_{v}(T)=\frac{2 h v^{2}}{c^{2}} \frac{1}{\mathrm{e}^{\frac{h v}{k T}}-1}$,

where $h$ denotes the Planck constant, $c$ - the speed of light, $k$ - the Boltzmann constant and $T$ is the temperature of the stellar galaxy component. The dust emission of galaxies $\left(v=10^{12}-10^{13} \mathrm{~Hz}\right.$, i.e. $\left.\lambda=30-300 \mu \mathrm{m}\right)$ was modeled with a modified black-body

$B_{v}^{\prime}(T)=v^{\gamma} B_{v}(T)$

where the parameter $\gamma=1.5$

Since we consider these models to be indicative only, we serached for the best-fit parameters of the black-body spectra by means of $\chi^{2}$ minimization, without taking errors into account. Since the black-body spectra provide a rather poor fit to the data, we found that using error information does not improve the 
K. Małek et al.: Star forming galaxies in the ADF-S

Table 6. Morphological and environmental properties of 47 ADF-S galaxies used for fitting SED models.

\begin{tabular}{|c|c|c|c|}
\hline $\begin{array}{c}\text { ID } \\
(\mathrm{ADFS})\end{array}$ & Type of source ${ }^{a}$ & Morphological type ${ }^{\mathrm{b}}$ & $\begin{array}{l}\text { Number of } \\
\text { counterparts }^{\mathrm{c}}\end{array}$ \\
\hline 1 & Galaxy/IRS/RS & $\mathrm{Sb}$ & 1 \\
\hline 2 & Galaxy in Pair of Interacting Galaxies/IRS/RS & $\mathrm{E}$ & 1 \\
\hline 3 & Galaxy/IRS/RS/other & $\mathrm{Sa}$ & 1 \\
\hline 4 & $\begin{array}{l}\text { Stardust Galaxy IR emission line up with } \\
\text { Super Star Cluster/IRS/RS/other }\end{array}$ & S0, SA0- pec: HII BCDG & 1 \\
\hline 5 & Galaxy/IRS/RS & $\mathrm{S}$ & 2 \\
\hline 6 & Galaxy/IRS & - & 4 \\
\hline 7 & Galaxy in Cluster of Galaxies/IRS & $\mathrm{S}^{1}$ & 1 \\
\hline 8 & Galaxy in Cluster of Galaxies/IRS & $\mathrm{Sb}^{1}$ & 2 \\
\hline 9 & Galaxy in Cluster of Galaxies & $\mathrm{Sb}^{1}$ & 3 \\
\hline 10 & Galaxy in Cluster of Galaxies/IRS/RS & S00 pec ${ }^{1}$ & 1 \\
\hline 11 & Galaxy in Cluster of Galaxies/IRS/RS & $\mathrm{SB}^{1}$ & 1 \\
\hline 12 & IRS & - & 1 \\
\hline 13 & Galaxy/IRS & - & 2 \\
\hline 14 & Galaxy/IRS/RS & - & 2 \\
\hline 15 & Galaxy/IRS & - & 3 \\
\hline 16 & IRS/Galaxy in Pair of Galaxies & - & 2 \\
\hline 17 & Interacting Galaxies/IR/RS & Sbc pec & 3 \\
\hline 18 & Galaxy/IRS & $\mathrm{S}$ & 1 \\
\hline 19 & Galaxy/IRS & - & 2 \\
\hline 20 & Galaxy/IRS & - & 1 \\
\hline 21 & Galaxy/IRS & SAB & 1 \\
\hline 22 & Galaxy/IRS & $\mathrm{S}$ & 2 \\
\hline 23 & Interacting Galaxies & cG (compact G), Chain of 3 galaxies & 1 \\
\hline 24 & Galaxy/IRS & $\mathrm{S}$ & 2 \\
\hline 26 & Galaxy & - & 2 \\
\hline 27 & IRS & - & 1 \\
\hline 29 & Galaxy/IRS & SOa & 1 \\
\hline 30 & IRS/Galaxy & - & 1 \\
\hline 31 & IRS/Galaxy & - & 1 \\
\hline 39 & Galaxy/IRS & So & 3 \\
\hline 41 & Galaxy/IRS & - & 2 \\
\hline 42 & Galaxy/IRS & - & 1 \\
\hline 43 & IRS/Galaxy & - & 1 \\
\hline 45 & Galaxy in Cluster of Galaxies/IRS & $\mathrm{Sbp}^{1}$ & 1 \\
\hline 46 & Galaxy/IRS & - & 1 \\
\hline 48 & Galaxy/IRS & - & 1 \\
\hline 49 & Galaxy & - & 2 \\
\hline 50 & Galaxy/IRS & S & 1 \\
\hline 51 & Galaxy/IRS/RS & SB & 1 \\
\hline 52 & IRS/Galaxy & - & 1 \\
\hline 56 & Galaxy/IRS & - & 5 \\
\hline 64 & Galaxy/IRS & $\mathrm{Sc}$ & 1 \\
\hline 65 & Galaxy in Cluster of Galaxies/IRS & $\mathrm{S}^{1}$ & 1 \\
\hline 69 & Galaxy/IRS & - & 2 \\
\hline 72 & Galaxy in Cluster of Galaxies/IRS & Sbb pec ${ }^{1}$ & 2 \\
\hline 73 & IRS & - & 1 \\
\hline 82 & Galaxy & - & 1 \\
\hline 103 & Galaxy in Cluster of Galaxies/IRS & $\mathrm{Sb}^{1}$ & 2 \\
\hline
\end{tabular}

Notes. ${ }^{(a)}$ The object classification: Galaxy means that it has been identified as a galaxy, IRS means that it has been previously identified as the infra-red source, RS means that it has been identified as the radio source, "other" means that it has been observed in some other (UV or X-ray) wavelengths. This column contains also the available information about the object's environment - whether it is an object from the cluster of galaxies (in this case it always applies to Abell S0463) or an interacting group of galaxies. ${ }^{(b)}$ The object's indicative morphological type, as provided by the NED. ${ }^{(c)}$ The number of counterparts found for each object in our 40" search range, which may be treated as a very crude indicator of the local environment of an investigated object. ${ }^{(1)}$ Dressler (1980a).

fitting, and in some cases even makes the black-body models more difficult to fit.

We found the best-fitting model parameters by minimizing the quantity

$\chi^{2}=\sum_{i=1}^{n} \frac{\left(v S_{v, i}-B_{v}^{\prime}(T)_{i}\right)^{2}}{B_{v}^{\prime}(T)_{i}}$, where $n$ is the number of data points, $v S_{v, i}$ is the measured flux, and $B_{v}^{\prime}(T)_{i}$ is the corresponding theoretical value given by a black-body or modified black-body model.

If the absolute difference between data and the best-fit was greater than $2 \sigma$, we repeated the fitting process, but without taking into account the point for which the relative difference was the highest, provided that at least three data points remained. The 
result of the rejection of the least closely fitting point in case of a poor fit can be seen e.g., in Fig. 13 for galaxy number 8: the data point in the B filter (the last point in the optical range) had to be removed to provide a reasonable fit.

Perhaps not coincidentally, the data points that are most often rejected from the fitting procedure are measurements in the optical B filter. These are usually measurements of the poorest quality. For instance, in the case of 4 galaxies (sources 7, 8 in Fig. 13, source 9 in Fig. 14, and source 45 in Fig. 17), these measurements, acquired from the SIMBAD database, originally come from the Dressler's catalog of galaxies in clusters (Dressler 1980b). In the original paper, they are described as the estimated total apparent visual magnitude and their accuracy is about one magnitude. They are obviously unsuitable for SED fitting. Other often rejected points, also in the B band (e.g. galaxy number 6 in Fig. 13), come from the APM survey (Maddox et al. 1990), whose photometry is known to be affected by systematic bias (e.g., Metcalfe et al. 1995; Bertin \& Dennefeld 1997).

Given the poor reliability of the stellar black-body spectra, we note that the "temperature" of the stellar component provides only a symbolic index of the hardness of the SEDs and not an absolute value of the temperature of their stellar population. The temperature is used here only as an internal comparison of the analyzed ADF-S sources.

We did not take into account dust attenuation in the fitting. For this kind of analysis, we would need to use a more sophisticated stellar spectral synthesis model to extract information about the dust attenuation assuming a certain dust attenuation curve (e.g., Mathis 1990; Calzetti et al. 1994, 2000). However, in addition to the rough approximation of stellar spectra by a black body, almost all our SEDs lack UV-optical photometry which hampers a precise determination of extinction. If we had a FIR/UV flux ratio, we would be able to estimate a correlation between FIR/UV flux ratio and luminosity from newly forming stars (Buat et al. 2005, 2007). Unfortunately, the UV photometry or distance of a source is only available for a small number of sources in our sample. Hence, we discuss a possible effect of dust attenuation only qualitatively.

As stated before, dust scatters and absorbs UV-optical light from stars and re-emits the energy in the IR. Since conventional attenuation curves of galaxies have a steep rise toward the UV, dust attenuation causes the reddening of galaxy stellar spectra. This then causes a significant underestimation of stellar temperature by black-body fitting.

The best-fit values of temperature of stars and dust in the modeled galaxies are listed in the second and third column of Table 5. The histograms of these two temperatures for all 47 modeled galaxies are presented in Fig. 10. According to this measurement, both the median and mean temperature of dust is around $32 \pm 4 \mathrm{~K}$. In contrast, the median temperature of the stellar component is $2240 \pm 360 \mathrm{~K}$, while its mean is $2830 \pm 410 \mathrm{~K}$, the discrepancy between mean and median values being caused by the temperature distribution being far from Gaussian.

The inferred values of stellar temperatures are generally systematically too low for stars that emit significantly in the UV. As we mentioned above, the low estimated temperature of our sample could be partially due to the dust attenuation. Here the temperature value is used only to make an internal comparison of our sample.

The highest stellar temperature that we fitted was for a starburst blue compact dwarf NGC 1705. The dust temperature of this galaxy is also significantly higher than average. However, as can be seen in the fourth panel in Fig. 13, a simple black body provides a very poor fit for its stellar population.
The elliptical galaxy ESO 157-IGA040 is significantly warmer than the median stellar temperature, at $T_{*}=3060 \mathrm{~K}$, and its dust temperature is also relatively high, at $T_{\text {dust }} \approx 38 \mathrm{~K}$. As seen in the second panel of Fig. 13, black-body models seem to describe the data well. The activity of this elliptical galaxy should be related to it being a member of a pair of interacting galaxies.

Even if 15 spiral and five lenticular galaxies provide a rather poor statistical sample, we attempted some simple comparison between these two groups.

Rather surprisingly, spiral and lenticular galaxies in our sample seem to have very similar properties, and spirals typically have even lower dust and stellar temperatures than lenticulars. The mean dust temperature of spirals $(\sim 30 \pm 5 \mathrm{~K})$ is $5 \mathrm{~K}$ lower than that of lenticulars $(\sim 35 \pm 17 \mathrm{~K})$, while the average temperature of a stellar component in spirals is $880 \pm 200 \mathrm{~K}$ lower than in the case of lenticulars. The difference in the dust temperatures is mainly caused by the two outliers with the highest dust temperatures being lenticulars and a galaxy with the lowest fitted dust temperature being a spiral. However, spectra of these objects seem to be fitted reasonably well by the black-body models. Consequently, when considering the median values, the difference is less pronounced: the median dust temperature is $29 \pm 4 \mathrm{~K}$ for lenticulars and $28 \pm 4 \mathrm{~K}$ for spirals. The difference between median stellar temperatures for spirals and lenticulars is also significant $(570 \pm 130 \mathrm{~K})$ but smaller.

Of course, given the small number statistics, this difference between spiral and lenticular galaxies is not significant. It might be interpreted as lenticulars that are detected in FIR are particularly active in star formation at least at the same level as average spirals. However, it is more plausible that the simplest models are not particularly accurate in reproducing the dust and stellar emission of this type of galaxy.

\subsubsection{Dale and Helou model}

As mentioned above, a modified black-body model provides a poor fit to the MIR part of dust spectrum. To fit the dust emission of our galaxies across wide range of wavelengths between 3 and $1100 \mu \mathrm{m}$, we applied a model proposed by Dale \& Helou (2002). This was developed to model a broad range of interstellar environments in normal star-forming galaxies at different heating intensity levels. In this model, the local dust SED is given by a power-law distribution

$\mathrm{d} M_{\mathrm{d}}(U) \propto U^{-\alpha_{\text {SED }}} \mathrm{d} U$,

where $M_{\mathrm{d}}(U)$ is the mass of dust heated by a radiation field $U$, and $\alpha_{\mathrm{SED}}$ is a parameter that represents the relative contributions of active and quiescent regions from different galaxies. Values of $\alpha_{\text {SED }} \leq 1$ represent actively star-forming galaxies. The lower the value of $\alpha_{\mathrm{SED}}$, the more actively star forming a galaxy is. More quiescent and cooler galaxies have $1<\alpha_{\mathrm{SED}}<2.6$. The case of $\alpha_{\mathrm{SED}}=2.6$ corresponds to the most quiescent dust model. Values of $\alpha_{\text {SED }}$ between 2.6 and 4 are fitted for galaxies where the FIR emission peak appears at even longer wavelengths than the most quiescent well studied galaxies (Dale et al. 2005). This region is still not very well examined but appears to correspond to the coolest and most quiescent galaxies.

If sufficient data are available, it is possible to calculate the expected value of $\alpha_{\text {SED }}$ e.g., from IRAS $f_{v}(60 \mu \mathrm{m})$ and $f_{v}(100 \mu \mathrm{m})$ measurements (Moshir et al. (1990); this method was presented e.g. in Dale \& Helou 2001). However, in our case, a relatively small amount of data and because we explore a rather 

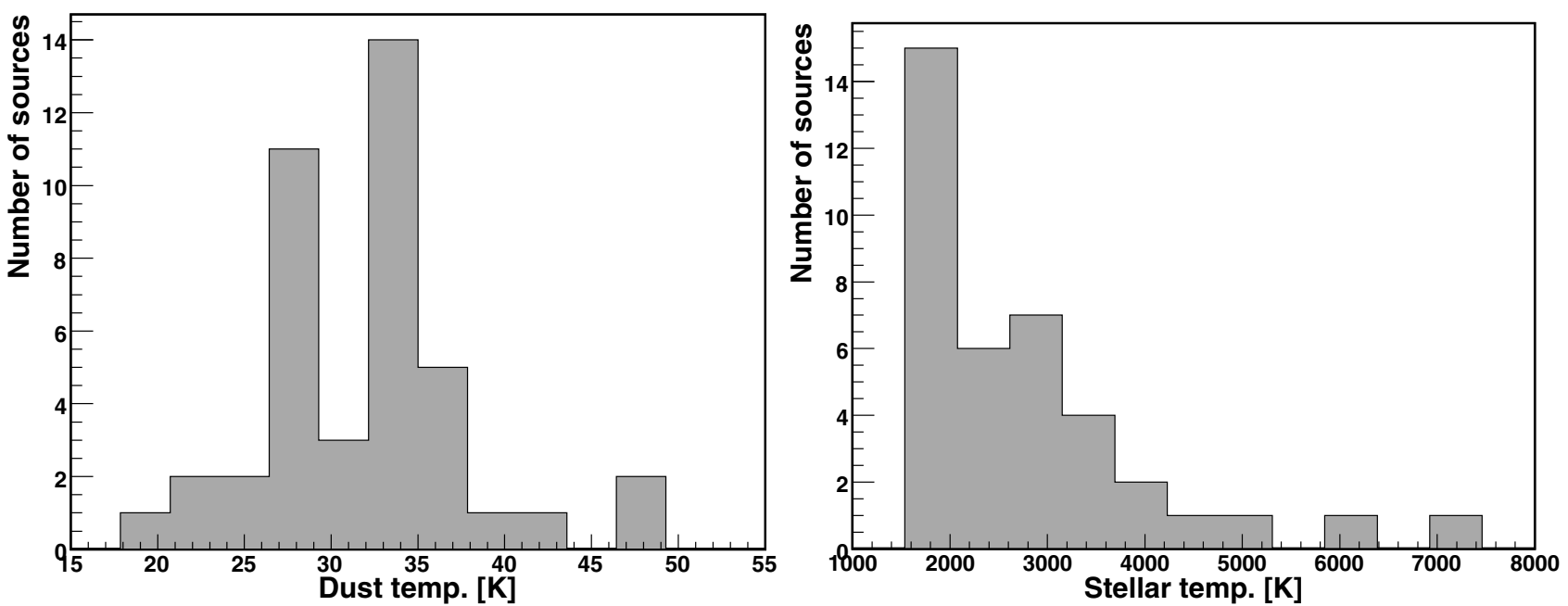

Fig. 10. Histograms of temperatures of dust and stellar components of galaxies in the best-fit modified blackbody (dust, left panel) and blackbody (stars, right panel) models.

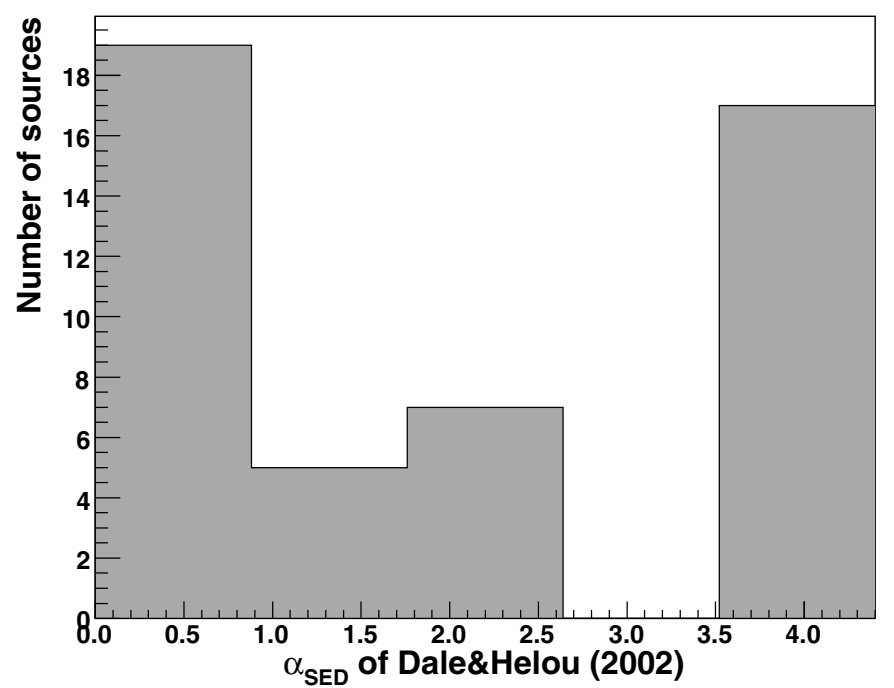

Fig. 11. The histogram of the parameter $\alpha$ of the model by Dale \& Helou (2002).

poorly known regime of FIR, prompted us to use minimization $\chi^{2}$-fitting. We took into account uncertainties in the photometric measurements. To compare our data points to the models effectively, the modeled spectra were convolved with the AKARI, IRAS, and Spitzer photometric bands. Using a grid of models with all the parameters available, we searched for the best-fit model with a minimal $\chi^{2}$ defined as

$\chi^{2}=\sum_{i=1}^{n} \frac{\left(\left(v S_{v, i}+K\right)-F_{\nu, i}\right)^{2}}{\sigma_{v, i}^{2}}$,

where $n$ is a number of points, $K$ is a normalization constant, used as a free parameter, $v S_{v, i}$ is a measured flux, $F_{v, i}$ is a corresponding theoretical value of flux from the dust model, and $\sigma_{v, i}$ is the corresponding measurement error. The resulting $\alpha_{\mathrm{SED}}$ for all modeled galaxies are listed in Col. 4 of Table 5.

Histogram of $\alpha_{\mathrm{SED}}$, presented in Fig. 11, shows that the distribution of $\alpha_{\mathrm{SED}}$ is quite discontinuous. Most of the analyzed sources have extreme values of $\alpha_{\text {SED }}$ lower than 0.9 ( 19 sources) or higher than 3.5 (17 sources). We found seven galaxies with values of $\alpha_{\text {SED }}$ between 1.7 and 2.,1 and five with values $\alpha_{\text {SED }}$ between 0.9 and 1.7. No galaxies were fitted by models with $\alpha_{\text {SED }}$ between 2.2 and 3.5.

Among galaxies with known morphological types, $80 \%$ of lenticular galaxies were fitted as the warmest, actively starforming galaxies with $\alpha_{\mathrm{SED}}<1$ and only one of them, 2MASX J04292360-5330114, was placed in a different regime, as the most quiescent galaxy with $\alpha_{\mathrm{SED}}=4$. No lenticular galaxy was found to be in the $\alpha_{\mathrm{SED}}=1-2.6$ regime.

The elliptical and 4 of the spiral galaxies were classified as cool and quiescent. One of the compact galaxies, the starburst blue compact dwarf NGC 1705 , and $60 \%$ of the spiral galaxies, were classified as warm and actively star forming. ESO 157-51, a second compact galaxy, and the remaining $40 \%$ of spiral galaxies were assigned to the most poorly examined and, in all probability, the most quiescent regime with $\alpha_{\mathrm{SED}}>3$. This suggests that the peak of their dust emission is yet to be located at longer wavelengths.

We compared our conclusions with the results of Dale et al. (2005), who constructed SEDs for 71 nearby galaxies in the SINGS sample (Kennicutt et al. 2003) across the range of $\lambda$ from $1 \mu \mathrm{m}$ to $850 \mu \mathrm{m}$. Among them, there were five elliptical galaxies with $\alpha_{\text {SED }}$ between 1.32 and 2.14 and one with $\alpha_{\text {SED }}=4$. This range of $\alpha_{\mathrm{SED}}$ indicates that all elliptical galaxies in the SINGS sample are cold and quiescent. The only elliptical galaxy in our sample seems to belong to the same type. This is expected, inconsistent with the results of the simple black-body fitting, which may be explained by the insufficiency of the latter technique.

In the sample used by Dale et al. (2005) there were also two lenticular galaxies, one of them warm and active in star formation $\left(\alpha_{\mathrm{SED}}=0.6\right)$ and the other cold $\left(\alpha_{\mathrm{SED}}=2.67\right)$. In this case, because of poor number statistics, it is difficult to make any comparison, but our modeling confirms that lenticulars can be very warm as well as extremely quiescent.

The only discrepancy concerns spiral galaxies. In the data used by Dale et al. (2005), 54 spiral galaxies were included and none was classified as actively star forming: 54\% of them were cold and quiescent with $\alpha_{\text {SED }}$ between 1.68 and 2.6 and the remaining $45 \%$ were described as even more quiescent with $\alpha_{\text {SED }}>2.6$. In our case, half of the spiral galaxies are very warm and active in star formation, there are no quiescent ones with $\alpha_{\mathrm{SED}}$ between 1.68 and 2.6 but the other half are spiral 


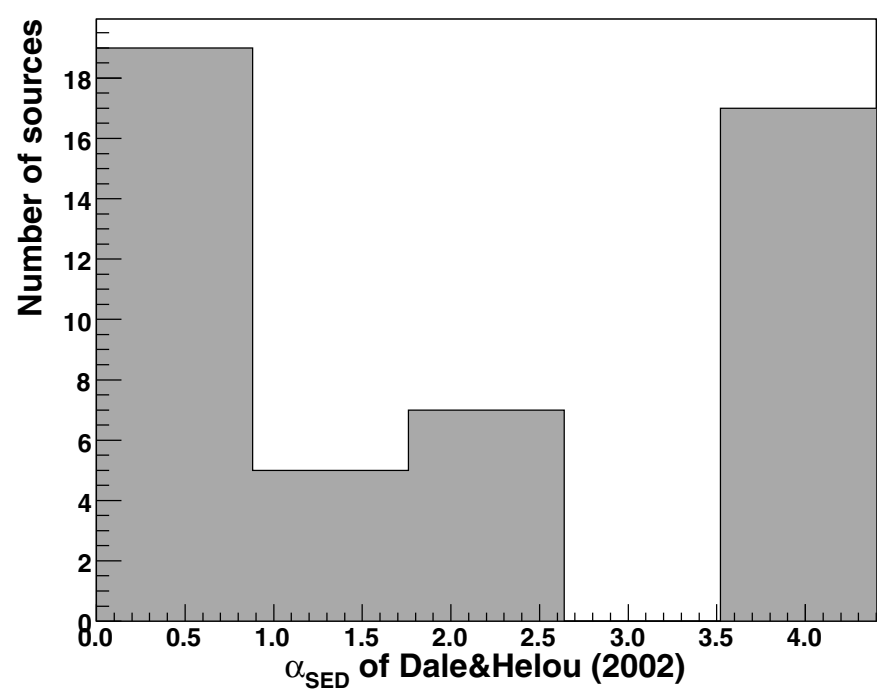

Fig. 12. The histogram of the amount of polycyclic aromatic hydrocarbons (PAH) in the dust of the analyzed galaxies, according to the model of Li \& Draine (2001).

galaxies with $\alpha_{\text {SED }}=4$, i.e., extremely quiescent. This finding is consistent with our sample being based on a genuine FIR (90 $\mu \mathrm{m})$ selection. On the one hand, this sample contains a high proportion of dusty IR galaxies, but on the other hand, it can also select galaxies that are quiescent because of its long wavelength coverage compared to IRAS-based studies (usually based on $60 \mu \mathrm{m}$ selected data).

\subsubsection{Li and draine model}

As yet another more refined model of the infrared emission from dust grains heated by starlight in galaxies, we chose the model proposed by Draine \& Li (2007), which is an improved version of Li \& Draine (2001). This model assumes that the dust heated by starlight consists of a mixture of amorphous silicate and carbonaceous grains. Each molecule has a wide size distribution ranging from molecules containing tens of atoms to large grains $\geq 1 \mu \mathrm{m}$ in diameter, according to Draine \& Li (2007), who calculated the emission spectrum for dust heated by the stellar light and parametrized this model using three sets of parameters: the fraction of total dust mass that the polycyclic aromatic hydrocarbon $(\mathrm{PAH})$ particles $\left(q_{\mathrm{PAH}}\right)$ contribute, the lower $\left(U_{\min }\right)$ and higher $\left(U_{\max }\right)$ cutoff to the starlight intensity distribution, and the fraction of dust heated by the starlight $(\gamma)$.

As before, the modeled spectra were first convolved with the AKARI, IRAS, and Spitzer photometric bands. We fitted the models of Draine \& Li (2007) using a $\chi^{2}$ test and including as in case of the Dale \& Helou model - information about the photometric errors.

Draine \& Li (2007) calculated the parameters directly from Spitzer data which was impossible in our case, but may be a part of a future project. The resulting values of $q_{\mathrm{PAH}}$ are listed in Col. 7 of Table 5. The histogram of $q_{\mathrm{PAH}}$, shown in Fig. 12, has peaks around three particular values $q_{\mathrm{PAH}}=0.75,2.37$, and 4.58.

Spectra presented by Draine \& Li (2007) correspond to 11 dust models with $q_{\mathrm{PAH}}$ ranging from $0.1 \%$ to $4.58 \%$. The lowest value of $q_{\mathrm{PAH}}$ corresponds to the Small Magellanic Cloud (SMC) model $q_{\mathrm{PAH}}=0.75 \%, 1.49 \%$, and $2.37 \%$ correspond to the Large Magellanic Cloud (LMC), and the remaining 7 models are related to Milky Way (MW)-like galaxies. From 47 modeled
ADF-S sources $81 \%$ were identified as LMC-like galaxies and $9 \%$ corresponded to MW-type galaxies. No SMC-like galaxy was found. For the entire sample, the median is $q_{\mathrm{PAH}}=2.37 \%$.

All five lenticular galaxies, as well as both compact dwarfs in our sample are best-fit by LMC-like models with values of $q_{\text {PAH }} \leq 2.37$. The SEDs of the majority $(87.5 \%)$ of spiral galaxies are also most closely fitted by LMC-like models, while for the reminder MW-like models are applied. The optimal value of $q_{\text {PAH }}$ for spiral galaxies is $3.9 \%$. Then, spiral galaxies tend to be larger and richer in $\mathrm{PAH}$, but the difference from similar properties of lenticulars is not statistically significant. The elliptical galaxy has the highest $q_{\mathrm{PAH}}=4.55 \%$ among modeled galaxies and is classified as a MW-like galaxy. The discrepant conclusions about this particular galaxy probably reflect its more complex structure and processes, which may be related to its interactions with another galaxy.

\section{Conclusions}

In this paper, we have presented a catalog of counterparts to ADF-S $90 \mu \mathrm{m}$ sources detected at the $>6 \sigma$ level. We found counterparts for 545 of 1000 sources from the analyzed catalog. We discussed the properties of these sources and attempted to derive conclusions about the average properties of the sample.

The point source ADF-S catalog itself appeared to contain quite reliably determined positions, most of its counterparts being located at an angular distance significantly smaller than the nominal resolution of the FIS detector. A small number of counterparts detected at larger angular distances may possibly be the effect of contamination. In the position determination, we observe a small (on the order of $4^{\prime \prime}$ ) but systematic bias that depends on the location in the ADF-S. These small systematic errors should be taken into account in future work on these data.

We conclude that FIR sources detected in the ADF-S are mostly nearby galaxies. This conclusion is consistent, first of all, with the source number counts in the FIR, but also a large number of bright optical counterparts and the redshift distribution of counterparts. It should be noted, however, that the completeness of the identified sample, close to $100 \%$ for sources brighter than $0.1 \mathrm{Jy}$, declines rather steeply for fainter sources, to $55 \%$ for the entire $10 \sigma$ catalog.

The population of identified galaxies appears surprisingly normal, similar to that expected for local optically bright galaxies. The main differences are:

a) a significantly lower percentage of elliptical galaxies, which can be explained by them being less dusty than other galaxies;

b) a much higher percentage of peculiar galaxies, which can be attributed to the strong star-forming activity of these objects, related to intergalactic interactions, and hence stronger radiation of cold dust in them;

c) a detected fraction of lenticular galaxies (all in the cluster of galaxies Abell S0463) that is practically the same as expected in the optically bright galaxy population. This suggests that these galaxies contain a significant amount of cold dust and is consistent with more complex models of their formation than simple secular evolution.

The estimated source confusion is higher than $34 \%$ of the number of identified sources. This implies that removal of the effect of source confusion from the IR flux measurements and a proper estimation of the local environment of the FIR-bright galaxies will require dedicated observations of their closest neighborhood. 
K. Małek et al.: Star forming galaxies in the ADF-S
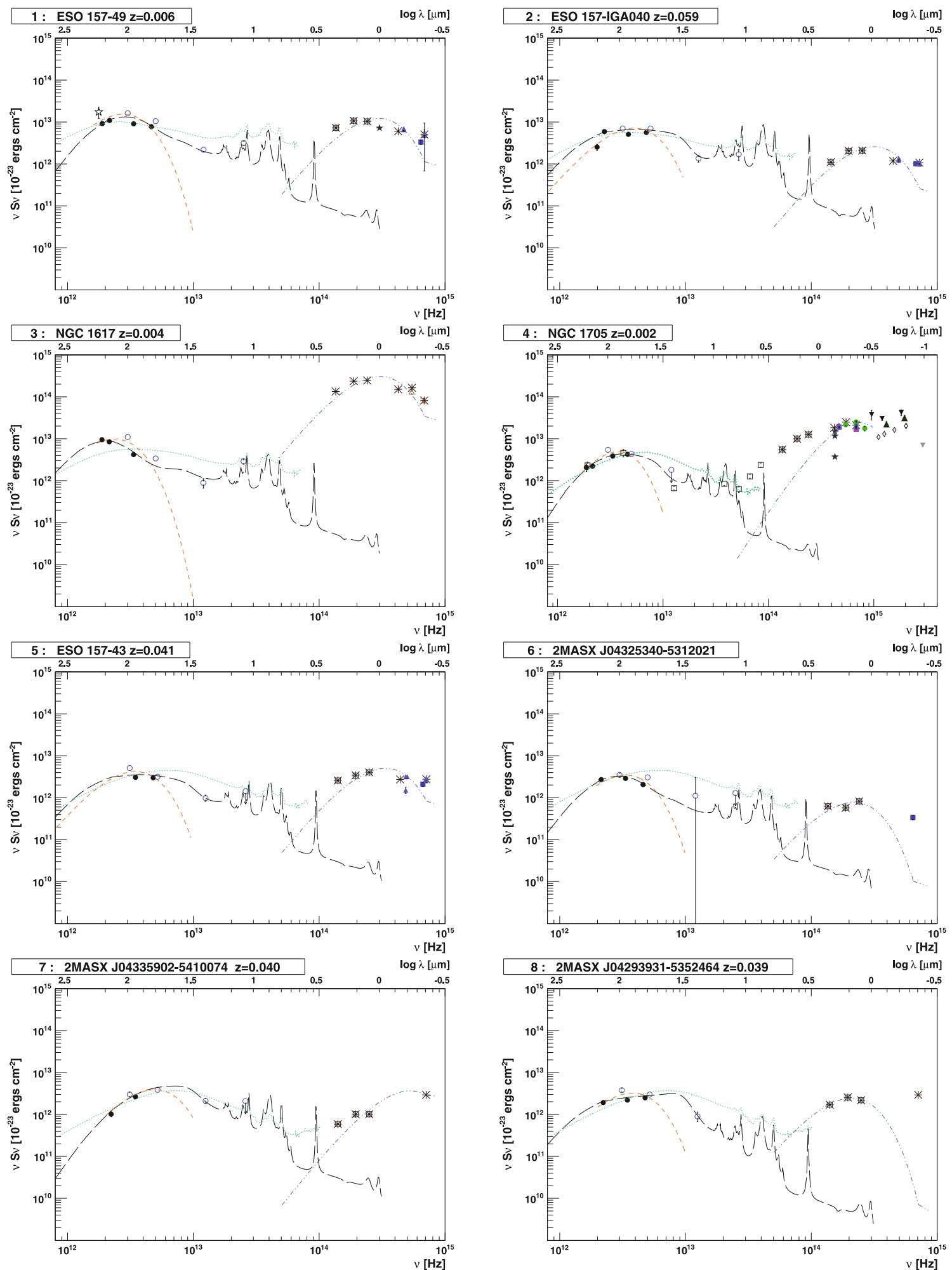

Fig. 13. The SEDs of ADF-S galaxies with the best photometry and available data from other catalogs. The data points from AKARI deep field south (full circles), 2MASS (open squares), SIMBAD database (eight pointed stars), IRAS (open circles), ESO/Uppsala (full triangles), APM (full squares), RC3 (full triangles), ISOPHOT (five pointed stars), Siding Spring Observatory (five pointed stars), GALEX (full triangles), HIPASS catalogue (full circles), Palomar/Las Campanas Imaging Atlas of Blue Compact Dwarf Galaxies (full squares), IUE (open diamonds), Spitzer (open squares), FUSE (upside-down light triangles) and UV: 1650, 2500, 315 (upside-down dark triangles) were fitted by three different models of dust emission: modified blackbody (short-dashed line) model of Dale \& Helou (2002) (dotted line), model of Li \& Draine (2001) (long-dashed line) and stellar emission: modified blackbody (dot-dot-dashed line). SEDs of galaxies with a given redshift (objects number 1, 2, 3, 4, 5, 7, 8) are fitted after shifting to the rest frame and presented in the rest frame. Galaxy number 6 , whose redshift is not known, is shown in the observed frame. 
A\&A 514, A11 (2010)
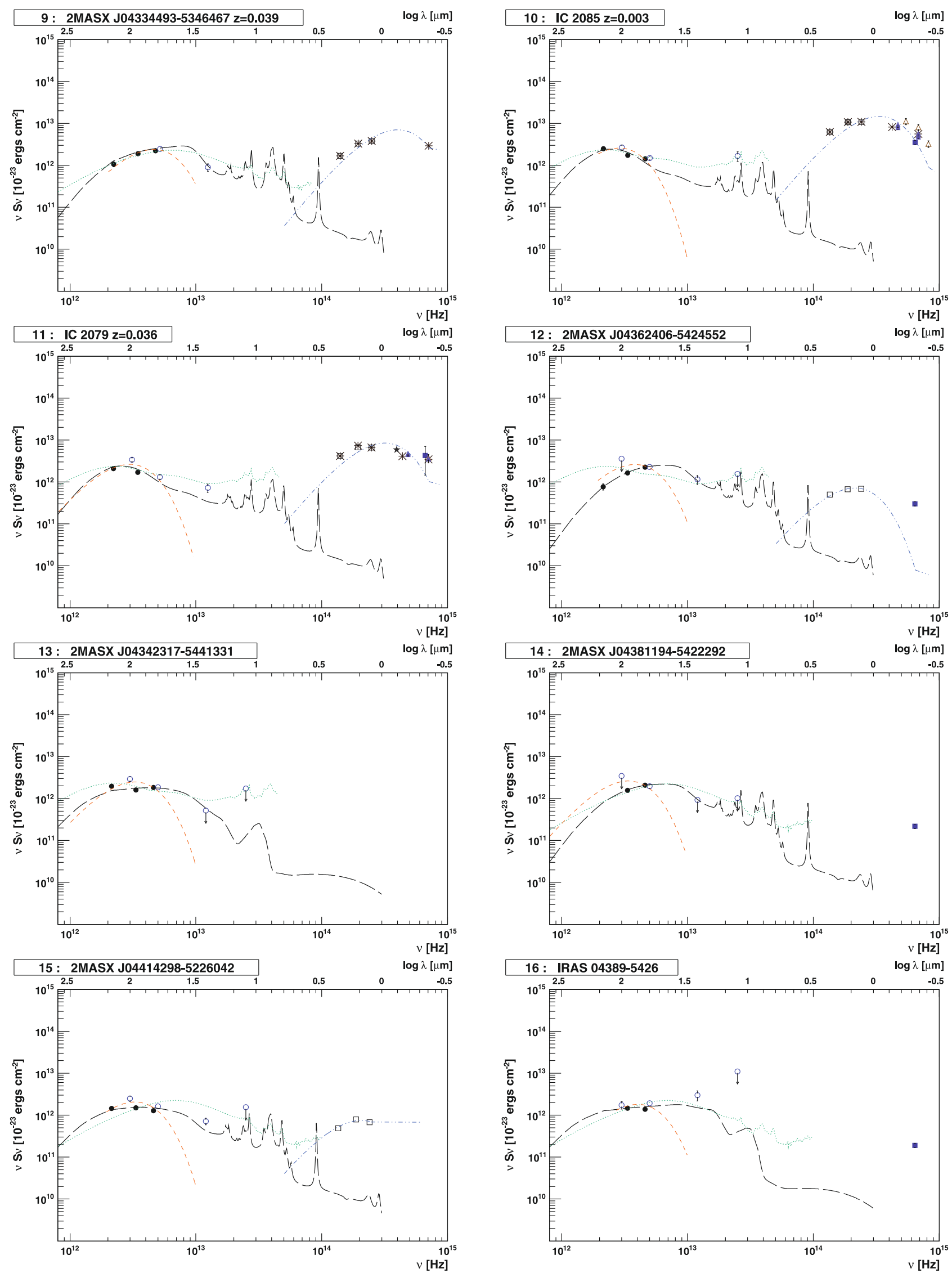

Fig. 14. Next 8 SEDs of ADF-S galaxies, where symbols are as in Fig. 13. SEDs of galaxies with a given redshift (objects number 9, 10, 11) are fitted after shifting to the rest frame and presented in the rest frame. The remaining objects are shown in the observed frame. 
K. Małek et al.: Star forming galaxies in the ADF-S
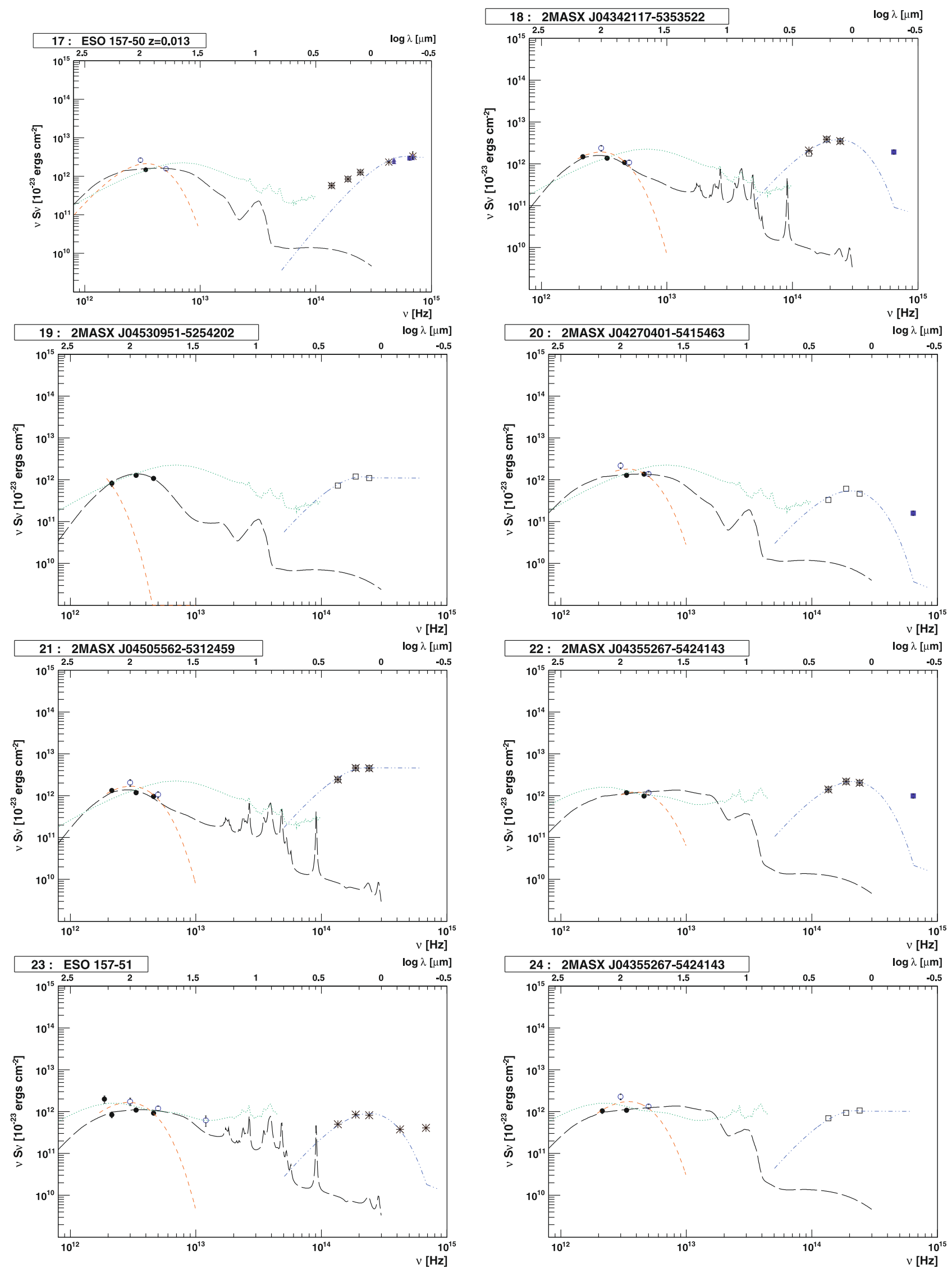

Fig. 15. Next 8 SEDs of ADF-S galaxies, where symbols are as in Fig. 13. SED of a galaxy number 17, for which the redshift is known, is fitted after shifting to the rest frame and presented in the rest frame. The remaining objects are shown in the observed frame. 
A\&A 514, A11 (2010)
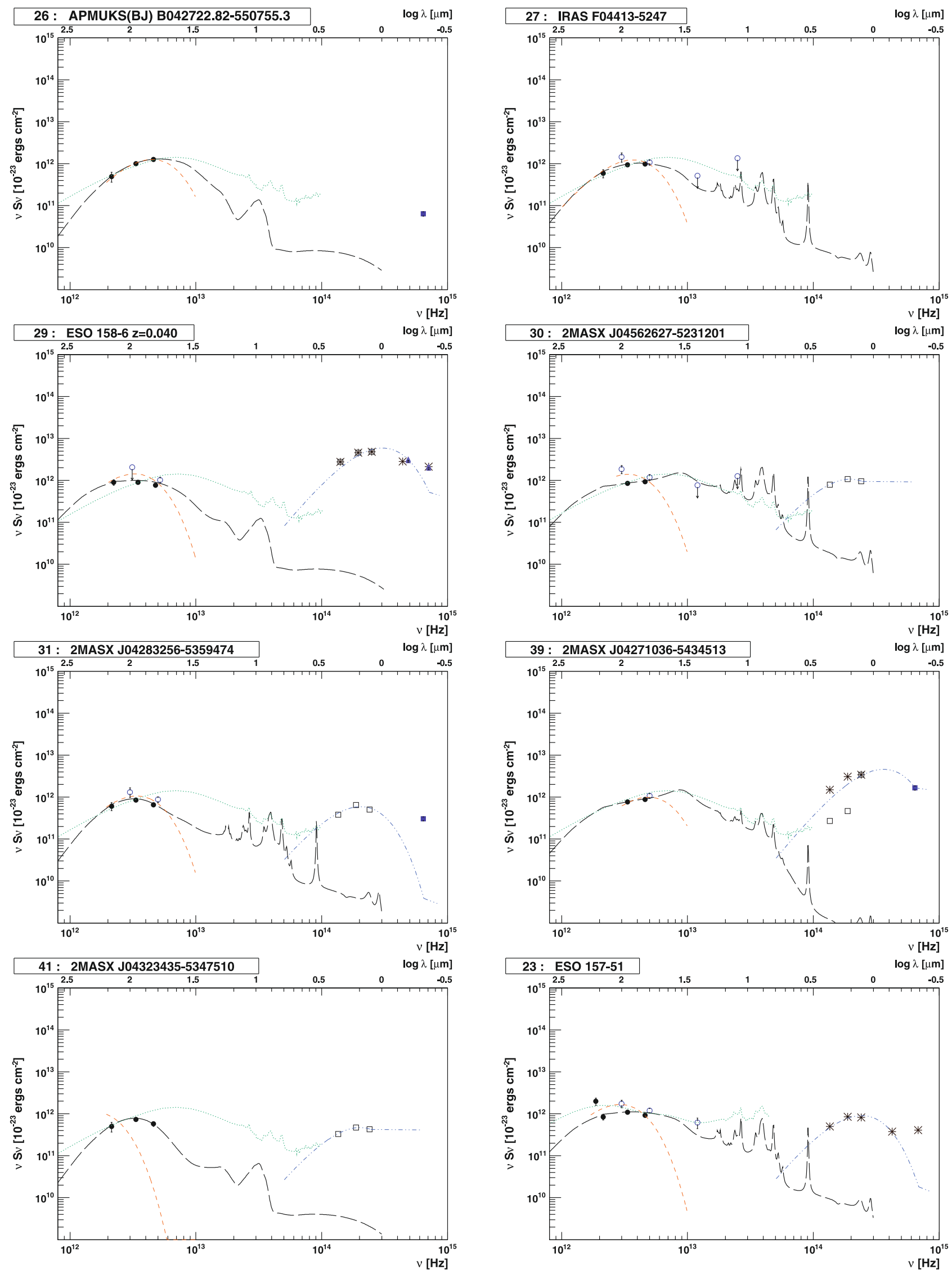

Fig. 16. Next 8 SEDs of ADF-S galaxies, where symbols are as in Fig. 13. SED of a galaxy number 29, for which the redshift is known, is fitted after shifting to the rest frame and presented in the rest frame. The remaining objects are shown in the observed frame. 
K. Małek et al.: Star forming galaxies in the ADF-S
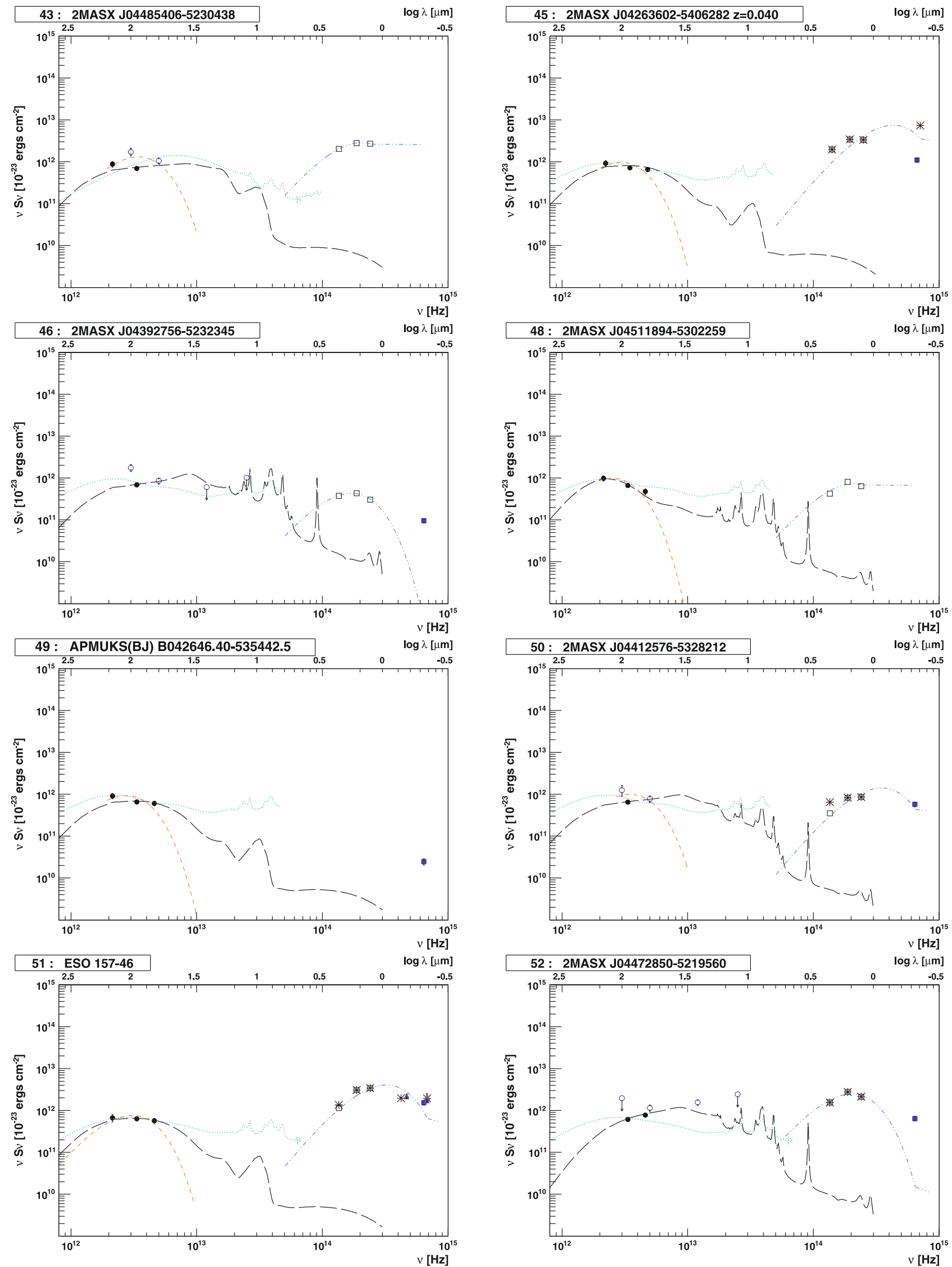

Fig. 17. Next 8 SEDs of ADF-S galaxies, where symbols are as in Fig. 13. SED of a galaxy number 45, for which the redshift is known, is fitted after shifting to the rest frame and presented in the rest frame. The remaining objects are shown in the observed frame. 
A\&A 514, A11 (2010)
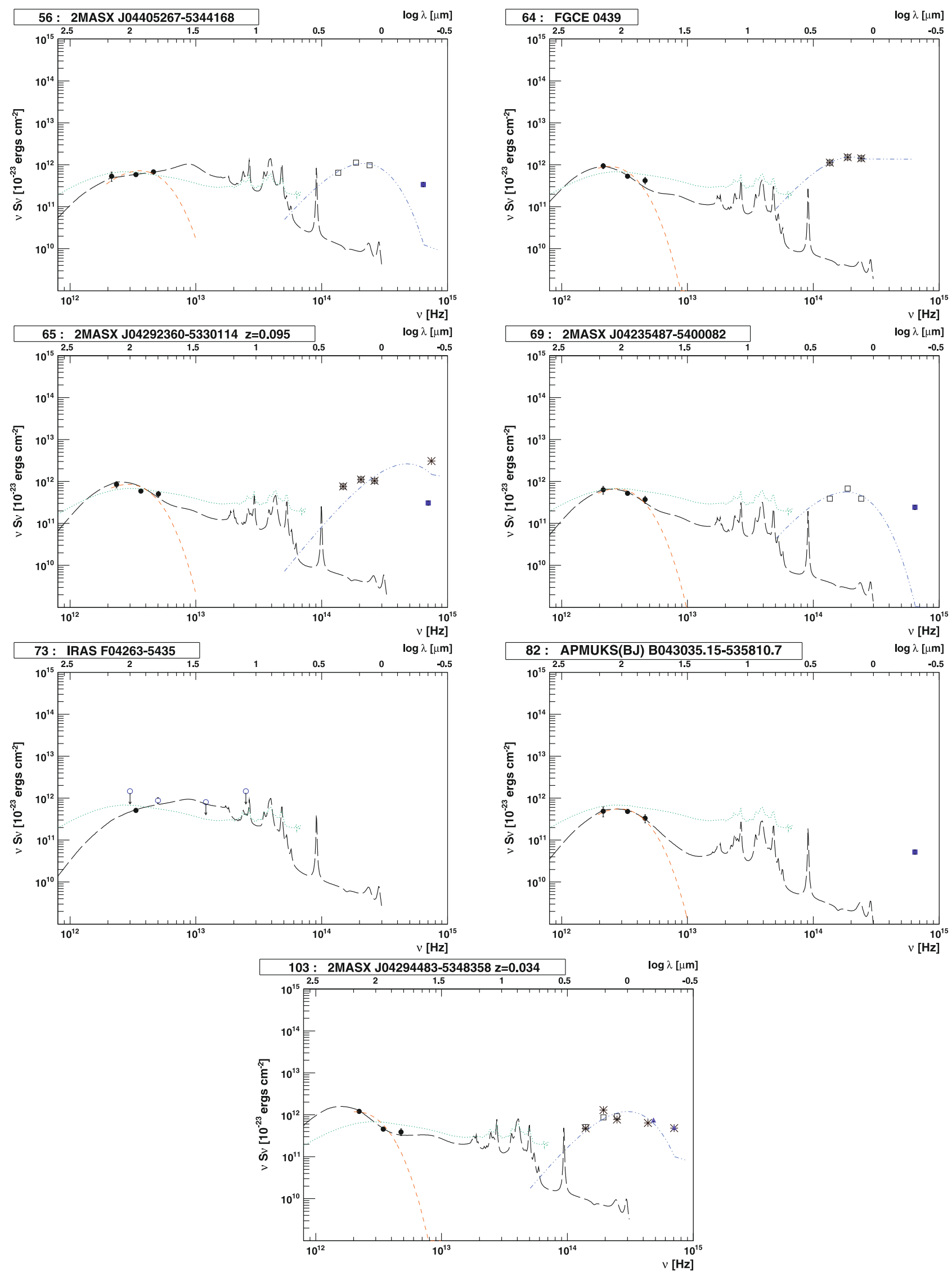

Fig. 18. Next 7 SEDs of ADF-S galaxies, where symbols are as in Fig. 13. SEDs of galaxies number 65 and 103, with known redshifts, are fitted after shifting to the rest frame and presented in the rest frame. The remaining objects are shown in the observed frame. 
The SEDs of the identified sources exhibit a variety of properties. In the first approach, the examined galaxies seem to be either extremely quiescent or very active in star formation. The lenticular galaxies usually belong to the actively star-forming group. The analysis suggests that to reproduce the FIR properties of otherwise normal galaxies in our sample, new updated models should be developed.

Acknowledgements. We thank the anonymous referee for her/his very careful reading of the manuscript and giving useful comments which significantly improved the clarity of this paper. This work is based on observations with AKARI, a JAXA project with the participation of ESA. This research has made use of the NASA/IPAC Extragalactic Database (NED) which is operated by the Jet Propulsion Laboratory, California Institute of Technology, under contract with the National Aeronautics and Space Administration, and the SIMBAD database, operated at CDS, Strasbourg, France We thank Misato Fukagawa for sending the information about Vega-like star candidates. This work has been supported in part by the Polish Astroparticle Physics Network. AP was financed by the research grant of the Polish Ministry of Science PBZ/MNiSW/07/2006/34A. TTT has been supported by Program for Improvement of Research Environment for Young Researchers from Special Coordination Funds for Promoting Science and Technology, and the Grant-in-Aid for the Scientific Research Fund (20740105) commissioned by the Ministry of Education, Culture, Sports, Science and Technology (MEXT) of Japan. TTT has been partially supported from the Grand-in-Aid for the Global COE Program "Quest for Fundamental Principles in the Universe: from Particles to the Solar System and the Cosmos" from the MEXT.

\section{References}

Abell, G. O., Corwin, H. G. Jr., \& Olowin, R. P. 1989, ApJS, 70, 1

Abadi, M. G., Moore, B., \& Bower, R. G. 1999, MNRAS, 308, 947

Barway, S., Wadadekar, Y., Kembhavi, A. K., et al. 2009, MNRAS, 394, 1991

Bekki, K. 1998, ApJ, 502, L133

Bertin, E., \& Dennefeld, M. 1997, MNRAS, 317, 43

Buat, V., Iglesias-Páramo, J., \& Seibert, M. 2005, ApJ, 619, L51

Buat, V., Takeuchi, T. T., Iglesias-Páramo, J., et al. 2007, ApJS, 173, 404

Calzetti, D., Kinney, A. L., \& Storchi-Bergmann, T. 1994, ApJ, 429, 582

Calzetti, D., Armus, L., \& Bohlin, R. C. 2000, ApJ, 533, 682

Cannon, J. M., Smith, J.-D. T., Walter, F., et al. 2006, ApJ, 647, 293

da Costa, L. N., Pellegrini, P. S., Davis, M., et al. ApJS, 1991, 75, 935

Dale, D. A., \& Helou, G. 2001, ApJ, 549, 215

Dale, D. A., \& Helou, G. 2002, ApJ, 576, 159

Dale, D. A., Bendo, G. J., Engelbracht, C. W., et al. 2005, ApJ, 633, 857

Dale, D. A., Gil de Paz, A., Gordon, K. D., et al. 2007, ApJ, 655, 863

Danks, A. C., Laustsen, S., \& van Woerden, H. 1979, A\&A, 73, 247

de la Torre, S., Le Fèvre, O., \& Arnouts, S. 2007, A\&A, 475, 443

de Souza, R. E., de Mello, D. F., \& Dos Anjos, S. 1997, A\&AS, 125, 329

de Vaucouleurs, G. 1963, ApJ, Suppl. 8, 31

de Vaucouleurs, G., de Vaucouleurs, A., Corwin, Jr. H. G., et al. 1991 (Berlin Heidelberg - New York: Springer-Verlag)

Dole, H., Lagache, G., Puget, J.-L., et al. 2006, A\&A, 451, 417

Draine, B. T., \& Anderson, N. 1985, ApJ, 292, 494

Draine, B. T., \& Li, A. 2001, ApJ, 551, 807

Draine, B. T., \& Li, A. 2007, ApJ, 657, 810

Dressler, A. 1980a, ApJS, 42, 565

Dressler, A. 1980b, ApJ, 236, 351

Dressler, A., \& Shectman, S. A. 1988, AJ, 95, 284

Doyle, M. T., Drinkwater, M. J., \& Rohde, D. J. 2005, MNRAS, 361, 34

Egan, M. P., Price, S. D., Kraemer, K. E., et al. 2003, VizieR Online Data Catalog, 5114
Ellis, R. S., Gray, P. M., Carter, D., et al. 1984, MNRAS, 206, 285 Fairall, A. P. 1984, MNRAS, 210, 69

Fox, A. J., Savage, B. D., \& Wakker, B. P. 2006, ApJS, 165, 229

Genzel, R., \& Cesarsky, C. J. 2000, ARA\&A, 38, 761

Gil de Paz, A., Madore, B. F., \& Pevunova, O. 2003, ApJS, 147, 29

Hubble, E. P. 1936, The Realm of the Nebulae (New Haven: Yale University

Press)

Kawada, M., Baba, H., Barthel, P. D., et al. 2007, PASJ, 59, S389

Kennicutt, R. C., Armus, L., Bendo, G., et al. 2003, PASP, 115, 928

Kinney, A. L., Bohlin, R. C., Calzetti, D., Panagia, N., \& Wyse, R. F. G. 1993, ApJS, 86, 5

Knapp, G. R., Guhathakurta, P., Kim, D.-W., et al. 1989, ApJS, 70, 329

Landy, S. D., \& Szalay, A. S. 1993, ApJ, 412, 64

Lauberts, A., \& Valentijn, E. A. 1989, The surface photometry catalogue of the ESO-Uppsala galaxies, Garching: European Southern Observatory

Lawrence, A., Rowan-Robinson, M., Ellis, R. S., et al. 2001, MNRAS, 326, 1333

Li, A., \& Draine, B. T. 2001, ApJ, 554, 778

Loveday, J., Peterson, B. A., Maddox, S. J., et al. 1996, ApJS, 107, 201

Maddox, S. J., Efstathiou, G., Sutherland, W. J., et al. 1990, 1990, MNRAS, 243 , 692

Mathis, J. S. 1990, ARA\&A, 28, 37

Mauch, T., Murphy, T., Buttery, H. J., et al. 2003, MNRAS, 342, 1117

Mathewson, D. S., \& Ford, V. L. 1996, ApJS, 107, 97

Mathewson, D. S., Ford, V. L., \& Buchhorn, M. 1992, ApJS, 81, 413

Metcalfe, N., Fong, R., \& Shanks, T. 1995, MNRAS, 274, 769

Metcalfe, N., Shanks, T., Campos, A., Fong, R., \& Gardner, J. P. 1996, Nature, 383,236

Moshir, M., Kopan, G., \& Conrow, T. 1990, BAAS, 22, 1325

Murakami, H., Baba, H., Barthel, P., et al. 2007, PASJ, 59, S369

di Nella, H., Paturel, G., Walsh, A. J., et al. 1996, A\&AS, 118, 311

Oliver, S., Waddington, I., \& Gonzalez-Solares, E. 2004, ApJS, 154, 30

Pahre, M. A., Ashby, M. L. N., Fazio, G. G., et al. 2004, ApJS, 154, 229

Paturel, G., Dubois, P., Petit, C., et al. 2002, LEDA, 0

Peebles, P. J. E. 1993, Principles of Physical Cosmology (Princeton: Princeton University Press)

Peebles, P. J. E., \& Groth, E. J. 1976, A\&A, 53, 131

Purcell, E. M. 1976, ApJ, 206, 685

Rifatto, A., Longo, G., \& Capaccioli, M. 1995, A\&AS, 114, 527

Roberts, M. S., \& Haynes, M. P. 1994, ARA\&A, 32, 115

Sandage, A. 1975, ApJ, 202, 563

Shirahata, M., Matsuura, S., Hasegawa, S., et al. 2009, PASJ, 61, 737

Sil'chenko, O. K., \& Afanasiev, V. L. 2004, AJ, 127, 2641

Skrutskie M. F., Cutri, R. M., \& Stiening, R. 2003, The 2MASS Extended sources (IPAC/UMass, 2003-2006), VizieR Online Data Catalog

Soifer, B. T., Houck, J. R., \& Neugebauer, G. 1987, ARA\&A, 25, 187

Soifer, B. T., Helou, G., \& Werner, M. 2008, ARA\&A, 46, 201

Takeuchi, T. T., Hirashita, H., Ishii, T. T., Hunt, L. K., \& Ferrara, A. 2003, MNRAS, 343, 839

Takeuchi, T. T., \& Ishii, T. T. 2004, ApJ, 604, 40

Takeuchi, T. T., Buat, V., \& Burgarella, D. 2005a, A\&A, 440, L17

Takeuchi, T. T., Buat, V., Iglesias-Páramo, J., Boselli, A., \& Burgarella, D. 2005b, A\&A, 432, 423

Takeuchi, T. T., Ishii, T. T., Nozawa, T., Kozasa, T., \& Hirashita, H. 2005c, MNRAS, 362, 592

Takeuchi, T. T., Ishii, T. T., Dole, H., et al. 2006, A\&A, 448, 525

Thomas, H.-C., Beuermann, K., Reinsch, K., et al. 1998, A\&A, 335, 467

van den Bergh, S. 1994, AJ, 107, 153

van den Bergh, S. 2009, ApJ, 702, 1502

Verma, A., Charmandaris, V., Klaas, U., Lutz, D., \& Haas, M. 2005, Space Sci. Rev., 119, 355

Wenger, M., Ochsenbein, F., Egret, D., et al. 2000, A\&AS, 143, 9

Wisotzki, L., Christlieb, N., Bade, N., et al. 2000, A\&A, 358, 77

Yoshii, Y., \& Takahara, F. 1988, ApJ, 326, 1 